\title{
Improvements in the orbitalwise scaling down of Perdew-Zunger self-interaction correction in many-electron regions
}

\author{
Yoh Yamamoto* Selim Romero, Tunna Baruah, and Rajendra R. Zopè \\ Department of Physics, University of Texas at El Paso, El Paso, Texas 79968
}

(Dated: April 10, 2020) 


\begin{abstract}
The Perdew-Zunger (PZ) method provides a way to remove the self-interaction (SI) error from density functional approximations on an orbital by orbital basis. The PZ method provides significant improvements for the properties such as barrier heights or dissociation energies but results in over-correcting the properties well described by SI-uncorrected semi-local functional. One cure to rectify the over-correcting tendency is to scale down the magnitude of SI-correction of each orbital in the many electron region. We have implemented the orbitalwise scaled down SI-correction (OSIC) scheme of Vydrov et al. [J. Chem. Phys. 124, 094108 (2006)] using the Fermi-Löwdin SI-correction method. After validating the OSIC implementation with previously reported OSICLSDA results, we examine its performance with the most successful non-empirical SCAN metaGGA functional. Using different forms of scaling factors to identify one-electron regions, we assess the performance of OSIC-SCAN for a wide range of properties: total energies, ionization potentials and electron affinities for atoms, atomization energies, dissociation and reaction energies, and reaction barrier heights of molecules. Our results show that OSIC-SCAN provides superior results than the previously reported OSIC-LSDA, -PBE, and -TPSS results. Furthermore, we propose selective scaling of OSIC (SOSIC) to remove its major shortcoming that destroys the $-1 / r$ asymptotic behavior of the potentials. The SOSIC method gives the highest occupied orbital eigenvalues practically identical to those in PZSIC and unlike OSIC provides bound atomic anions even with larger powers of scaling factors. SOSIC compared to PZSIC or OSIC provides more balanced description of total energies and barrier heights.
\end{abstract}

\title{
I. INTRODUCTION
}

The Kohn-Sham (KS) formulation[1, 2] of the density functional theory (DFT) is formally an exact approach to obtain the ground state energy of many electron system. It is by far the most widely used method for obtaining the electronic and structural properties of molecules and solids. Its practical applications require approximation to the exact exchange-correlation functional that is representative of the non-classical energy contributions. There is no systematic way to construct the functional, and a large number of approx-

*yyamamoto@utep.edu

$\dagger$ rzope@utep.edu 
imate functionals have been proposed and widely used. The exchange-correlation functional approximations are classified by Perdew and Schmidt[3] using an analogy to the Jacob's ladder wherein the functional approximation corresponds to the rungs of a ladder. The earliest functional approximation is the celebrated local spin density approximation (LSDA) [4] which forms the first rung of the ladder. The functionals with more complex ingredients such as density gradients, density Laplacians or Kohn-Sham orbitals belong to the higher rungs. Thus, the generalized-gradient approximation (GGA) [5], 6] goes beyond the LSDA by capturing non-homogeneity of density using density gradients corresponds to the second rung. Likewise, the third rung of the ladder corresponds to the meta-GGAs that use kinetic energy densities or density Laplacians while the fourth one corresponds to the hyper-GGA functionals, examples of which are the hybrid functionals[7] that include certain percentages of the Hartree-Fock (HF) exchange in the functional approximation. The functionals from the second to fourth rungs (GGAs, meta-GGAs, and hyper-GGAs) are widely used today in the molecular physics, solid state physics, and materials science. These functionals can describe many physical properties with sufficient accuracy. Their efficient numerical implementations, available in a large number of easy-to-use codes, have led to a proliferation of density functional based studies. One shortcoming of the majority of the density functional approximations (DFAs) mentioned above is that these approximations suffer from the selfinteraction error (SIE), which arises due to incomplete cancellation of the classical Coulomb interaction of an electron with itself by the approximate exchange-correlation term in the energy functional. In general, the modern semi-local functionals are sophisticated enough to provide a fairly accurate description of the equilibrium properties such as atomization energies but they fail to describe the properties such as transition states in chemical reactions, charge-transfer excitations, binding of an electron in some anions, dissociation of molecules. The SIE in these functionals is considered to be responsible for these failures [8, 9]. Indeed, the SIE is recognized to be a major limitation of DFAs that limits their universal usage 10 14]. In 1981, Perdew and Zunger[9] (PZ) proposed a method to eliminate SIE on an orbital by orbital basis. They applied this self-interaction correction (SIC) scheme to the LSDA which was the only known approximation at that time and found significant improvements in atomic properties. Their scheme later became known as PZSIC. Subsequent calculations on molecules in mid-eighties by the Wisconsin group[15-17] used localized orbitals to compute the self-interaction (SI) energies of molecules. Since then a number of studies have used 
SIC implementations [8, 18 67] to study atoms, molecules, and solids. It has been found in a number of studies that the PZSIC when used to compute thermochemical properties such as enthalpies of formation provides improvement over the LSDA functional, but the results are still not as accurate as those obtained using the GGAs. In particular, PZSIC when used with GGAs and meta-GGAs often worsens the results for thermochemical properties. It, however, does provide significantly improved results for properties such as reaction barriers and barrier heights where chemical bonds are stretched. This improvement is observed for all the DFAs (LSDA, GGA, and meta-GGAs). This conflicting performance of PZSIC for thermochemical properties and barrier heights is called the paradox of PZSIC[68], resolution of which was recently suggested by using the local scaling of the exchange-correlation and Coulomb energy densities[69]. A few schemes to rectify the over-correcting tendency of PZSIC have been proposed and examined. Jónsson and coworkers[70] scaled down the entire SIC contribution by $50 \%$, and reported improved performance in atomization energy. They also reported that using complex orbitals can improve the performance especially in case of LSDA. In 2006, Vydrov et al.[30] proposed a method that scales down SIC in the many electron region using an iso-orbital indicator weighted by the density of local orbital. To distinguish from the constant (global) scaling approach of Jónsson and coworkers, we shall hereafter call the orbital dependent scaling approach by Vydrov and coworkers as orbital scaling method. Vydrov and coworkers examined in detail the performance of various powers of scaling factor for correcting the SIE in the LSDA, Perdew, Burke, and Ernzerhof (PBE) [5], Tao, Perdew, Staroverov and Scuseria (TPSS) [71], and a hybrid of PBE with 25\% of exact exchange (PBEh) [72, 73] functionals. Subsequently, they also employed the orbital scaling to SIC to study the effect of scaled down SIC on the dissociation curves of $\mathrm{H}_{2}^{+}, \mathrm{He}_{2}^{+}$, $\mathrm{LiH}^{+}$, and $\mathrm{Ne}_{2}^{+}[74]$. They found that only the unscaled PZSIC consistently yielded qualitatively correct curves for all four systems[74]. Their orbital scaling approach to PZSIC is free from exact one- and nearly exact two-electron SI but still suffers many-electron SIE[74]. Thus, benefit of the orbital scaling was primarily limited to equilibrium properties.

Since the report of the work by Vydrov and coworkers, a number advances in the functional development have been reported. One important advance at the meta-GGA level is the development of strongly constrained and appropriately normed (SCAN) semilocal functional[75]. SCAN satisfies all 17 known exact constraints that a meta-GGA functional can satisfy. A number of studies reported in literature show that the SCAN functional 
provides improvement over other functionals for a wide variety of solid-state and molecular properties[76 78]. Recently, we investigated the performance of the SCAN functional and self-interaction corrected SCAN functional for a wide array of molecular properties and found that eliminating self-interaction errors improves the performance of SCAN for dissociation energies and barrier heights but it worsens the atomization energies[79]. The goal of the present work is multifold. We first want to examine the performance of orbital scaling when used with SCAN meta-GGA functionals for various electronic properties such as total atomic energies, ionization potentials, electron affinities, molecules atomization energies, reaction barrier heights, and dissociation and reaction energies. We also want to explore the use of alternative scaling factors in order to see if they provide any improvement over the scaling factor used by Vyrdov and coworkers. Finally, we want to explore if the orbital scaling approach can be modified by differentially scaling the SIC for orbitals to obtain even better all-around performance. We illustrate this idea by proposing a new orbital scaling scheme that preserves correct $-1 / r$ asymptotic behavior of the potentials for atoms. We also show that this new scaling scheme leads to significant improvements over the original orbital scaling approach for number of properties.

\section{THEORY}

The PZSIC method removes the SIE in the approximate density functionals by means of orbital-dependent corrections to the approximate functional as follows,

$$
E_{X C}^{P Z S I C-D F A}=E_{X C}^{D F A}\left[\rho_{\uparrow}, \rho_{\downarrow}\right]-\sum_{i \sigma}^{o c c}\left\{U\left[\rho_{i \sigma}\right]+E_{X C}^{D F A}\left[\rho_{i \sigma}, 0\right]\right\} .
$$

Here, $\rho_{i \sigma}$ is the density of the $i^{\text {th }}$ orbital of spin $\sigma$, and $U\left[\rho_{i \sigma}\right]$ and $E_{X C}^{D F A}\left[\rho_{i \sigma}, 0\right]$ are the selfCoulomb and the self-exchange-correlation energies. In their 1981 work, Perdew-Zunger [9] presented SIC calculations on the atoms using the orbital densities obtained from the KS orbitals. They also noted that delocalized nature of KS orbital for extended system will make the SIC non size-extensive. Subsequently, Pederson, Heaton and Lin[16, 17] implemented PZSIC using local orbitals and performed the first SIC calculation on molecules. These localized orbitals are obtained from the unitary transformation of the KS orbitals by minimizing the energy which results in the Pederson localization equations:

$$
\left\langle\phi_{j \sigma}\left|V_{j \sigma}^{S I C}-V_{i \sigma}^{S I C}\right| \phi_{i \sigma}\right\rangle=0
$$


Fermi-Löwdin orbital SIC (FLOSIC) [80] is a recently proposed approach to remove the SIE using the PZSIC methodology. In the FLOSIC, the optimal local orbitals, called FermiLöwdin orbitals (FLOs), are obtained by a unitary transformation that depends on positionlike variables such that unitary invariance of the total energy is ensured. The Fermi orbitals are constructed by introducing the Fermi orbital descriptor (FOD) positions [81 83]. Using the FOD positions $\mathbf{a}_{j}$, the KS orbitals $\psi_{i}$ are transformed into the Fermi orbitals $\phi_{j}$ as follows,

$$
\phi_{j}(\vec{r})=\frac{\sum_{\alpha}^{N} \psi_{\alpha}\left(\mathbf{a}_{j}\right) \psi_{\alpha}(\vec{r})}{\sqrt{\rho\left(\mathbf{a}_{j}\right)}} .
$$

Here, $N$ is the number of occupied orbitals. The localized Fermi orbitals $\left\{\phi_{i}\right\}$ are subsequently orthogonalized using Löwdin orthogonalization to obtain the FLOs. By finding the optimal FOD positions that minimize the total energy, we can find the solution of Eq. (1). The optimal positions of the FODs are obtained by minimizing the energy using either conjugate-gradient method or the L-BFGS algorithm[84].

As mentioned in Sec. I, the application of PZSIC worsens the description of equilibrium properties when used with semilocal functionals. To rectify the overcorrecting tendency of PZSIC, Vydrov and coworkers[30] scaled down the SIC in many-electron region using an orbital dependent scaling factor, $X_{i \sigma}^{k}=\int z_{\sigma}^{k}(\vec{r}) \rho_{i \sigma}(\vec{r}) d \vec{r}$. Here, $k$ is an integer and $z_{\sigma}(\vec{r})=\tau_{\sigma}^{W}(\vec{r}) / \tau_{\sigma}(\vec{r})$, where $\tau_{\sigma}(\vec{r})=\frac{1}{2} \sum_{i}\left|\nabla \psi_{i \sigma}(\vec{r})\right|^{2}$, being the non-interacting kinetic energy density, and $\tau_{\sigma}^{W}$ is the von Weizsäcker kinetic energy density. The iso-orbital indicator $z_{\sigma}$ is a function of position in space and interpolates between the uniform density region $\left(z_{\sigma}=0\right)$ and one-electron region, $z_{\sigma}=1$. Vydrov et al. [30] recommend $k \geq 3$ for the TPSS meta-GGA to preserve the correct fourth-order expansion in the limit of slow varying density. The same consideration should apply to SCAN meta-GGA. This way of scaling down PZSIC with an orbital dependent scaling factor will referred hereafter as OSIC.

The SIC energy in the OSIC approach of Vydrov et al. [30] is given by

$$
E^{O S I C}=-\sum_{i \sigma}^{o c c} X_{i \sigma}^{k}\left(U\left[\rho_{i \sigma}\right]+E_{X C}^{D F A}\left[\rho_{i \sigma}, 0\right]\right) .
$$

It is evident that OSIC reduces to PZSIC for $k=0$. In the $k \rightarrow \infty$ limit, Eq. (4) becomes zero with an exception of one electron system. For the one-electron systems, the scaling factor will be 1 for any integer $k$. The scaling factor $z_{\sigma}(\vec{r})=\tau_{\sigma}^{W}(\vec{r}) / \tau_{\sigma}(\vec{r})$, has the advantage that it vanishes in the uniform electron gas limit. It is not the only choice for the 
scaling factor in OSIC. A number of alternative choices can be made. Vydrov and Scuseria subsequently used the ratio of the orbital density to the total-spin density[32]. This does not require kinetic-energy densities and gave results comparable to those obtained using $z_{\sigma}(\vec{r})=\tau_{\sigma}^{W}(\vec{r}) / \tau_{\sigma}(\vec{r})$. In this work, we also explore use of two other scaling factors. The first one is the electron localization function (ELF) introduced by Becke and Edgecombe[85]. The ELF is commonly used for classifying chemical bonds and is defined as follows,

$$
\mathrm{ELF}=\frac{1}{1+\alpha^{2}}
$$

where $\alpha=\left(\tau-\tau^{W}\right) / \tau^{\text {unif }}$, and $\tau^{\text {unif }}=(3 / 10)\left(3 \pi^{2}\right)^{2 / 3} \rho^{5 / 3}$ is $\tau$ in the uniform-density limit. Using ELF in place of $z_{\sigma}$ partially satisfies the correct limits of the OSIC scaling factor. Although ELF $=1$ for the single orbital limit, ELF $=0.5$ in the uniform gas limit. Additionally, we also use $\beta$, another iso-orbital indicator defined as

$$
\beta=\frac{\tau-\tau^{W}}{\tau+\tau^{\text {unif }}}
$$

which has been used recently in construction of meta-GGA functionals [86]. Following how $\beta$ is used in functional design, we use $1-(2 \beta)^{2}$ as the alternative for $z_{\sigma}$ in $X_{i \sigma}^{k}$. Although this form can become negative, we included it nonetheless for comparison since it has the correct interpolation between the single orbital limit, $\lim _{\tau \rightarrow \tau^{W}}\left\{1-(2 \beta)^{2}\right\}=1$, and uniform-gas limit, $\lim _{|\nabla \rho| \rightarrow 0, \tau \rightarrow \tau^{\text {unif }}}\left\{1-(2 \beta)^{2}\right\}=0$ since $\tau^{W}$ becomes 0 . Thus, OSIC with this scaling factor also recovers the uniform gas limit as the SI-correction vanishes in this limit and OSIC reduces to the DFA.

Appraisal of the orbital scaling factor for various electronic properties [30] showed that orbital scaling requires different values of $k$ for different properties to obtain improved results. For example, excellent atomic energies are obtained for $k=4$, but $k=1$ or less is needed to obtain good estimates of reaction barrier heights. The orbitalwise scaling down of PZSIC leads to violation of some exact constraints satisfied in PZSIC. One such consequence is that it destroys the desirable correct $-1 / r$ behavior of the exchange-correlation potential of the PZSIC. The orbital scaling of Eq. (4) also provides the poor performance for many-electron SIC [4] compared to the original PZSIC. As the asymptotic behavior is important in many physical process such as electron delocalization or in an accurate description of the charge transfer process, a new scaling approach that preserves $-1 / r$ asymptotic of the potential can be formulated. We refer to this approach as selective-scaling-OSIC (SOSIC). The SOSIC 
correction to the energy in this approach is given by

$$
E^{S O S I C}=-\sum_{i \sigma}^{M} X_{i \sigma}^{k}\left(U\left[\rho_{i \sigma}\right]+E_{X C}^{D F A}\left[\rho_{i \sigma}, 0\right]\right)-\sum_{i \sigma}^{P} Y_{i \sigma}\left(U\left[\rho_{i \sigma}\right]+E_{X C}^{D F A}\left[\rho_{i \sigma}, 0\right]\right)
$$

where $M=N-P$, with $N$ being the total number of occupied electrons. $P$ is the number local orbitals corresponding to the electrons in the highest occupied orbital (HOO) shell. For example, for $A r$ atom $P=8$ as there are six electrons in the degenerate HOO shell that project onto $8 s p^{3}$ local orbitals. We set $Y_{i \sigma}=1$ to maintain the accurate asymptotic description of exchange-correlation potential. We shall show later that this SOSIC essentially preserves the accuracy of unscaled PZSIC HOO eigenvalues and leads to overall improvements of electronic properties in both the equilibrium cases as well as in stretched bond situations. The application of SOSIC requires identifying the FODs or FLOs that correspond to the HOO. This can be accomplished by finding the FLO which has maximal overlap with the KS HOO. We note that even though we used $Y_{i \sigma}$ to be unity its value can adjusted so that the negative of HOO eigenvalue matches with the exact experimental ionization potential. Adjusting the potential so that the magnitude of the HOO eigenvalue agrees with first ionization potential has been used previously in the context of fully analytic (grid free) Slater-Roothaan method[87]. In recent years, a similar procedure has been used to obtain the range separation parameters in many applications of range separated hybrids $\operatorname{method}[88-92]$.

We implemented the OSIC and SOSIC method in the FLOSIC code[93, 94] that is based on UTEP-NRLMOL. The Porezag-Pederson NRLMOL basis set [95] which is roughly similar to quadruple zeta quality basis was used. FLOSIC uses a variational mesh[96] that provides efficient numerical integration. The SCAN meta-GGA[75] was recently implemented[79] in the FLOSIC code. We used very dense mesh tailored for SCAN that provides energy convergence with respect to the radial mesh within $10^{-8} \mathrm{Ha}$ accuracy[79]. For calculations of anions, in addition to NRLMOL extra basis sets, long range s, p, and d single Gaussian orbitals are used where their exponents $(\beta)$ are extrapolated from $N^{\text {th }}$ basis of a given system using a relation as $\beta(N)^{2} / \beta(N-1)$. This inclusion of additional diffuse exponents was suggested by Withanage et al. [97] for giving better descriptions of the extended nature 
of the anions. The full Hamiltonian in the OSIC is given by

$$
\begin{aligned}
H_{i}= & -\frac{1}{2} \nabla^{2}+v(\vec{r})+\int \frac{\rho\left(\overrightarrow{r^{\prime}}\right)}{\left|\vec{r}-\overrightarrow{r^{\prime}}\right|} d \overrightarrow{r^{\prime}}+v_{X C}^{D F A}\left(\left[\rho_{\uparrow}, \rho_{\downarrow}\right], \vec{r}\right)-X_{i}^{k}\left(\int \frac{\rho_{i}\left(\overrightarrow{r^{\prime}}\right)}{\left|\vec{r}-\overrightarrow{r^{\prime}}\right|} d \overrightarrow{r^{\prime}}+v_{X C}^{D F A}\left(\left[\rho_{i}, 0\right], \vec{r}\right)\right) \\
& -z_{\sigma}^{k}(\vec{r})\left(U\left[\rho_{i}(\vec{r})\right]+E_{X C}^{D F A}\left[\rho_{i}(\vec{r}), 0\right]\right)-\sum_{m}\left(U\left[\rho_{m}(\vec{r})\right]+E_{X C}^{D F A}\left[\rho_{m}(\vec{r}), 0\right]\right) \frac{\partial X_{m}^{k}}{\partial \rho(\vec{r}) .}
\end{aligned}
$$

In the present implementation, we ignore the last two terms which arise due to variation of scaling factors in constructing the Hamiltonian. The calculations are performed selfconsistently using Jacobi updates [98] similar to earlier reported FLOSIC calculations [5759, 63 65, 67, 79, 97] but they are not full variational due to the neglected terms. We assessed the importance of the neglected terms by comparing our OSIC results with those of Vydrov and coworkers for the LSDA functional and obtained essentially the same results for various electronic properties studied here. For instance, using the OSIC-LSDA with $k=1$, mean absolute error (MAE) per electron of total energies for $\mathrm{Li}-\mathrm{Ar}$ is $0.004 \mathrm{Ha}$ in both methods, and MAEs for AE6 and BH6 are 18.0 and $3.3 \mathrm{kcal} / \mathrm{mol}$ with our implementation whereas Vydrov et al. obtained 21.0 and $3.5 \mathrm{kcal} / \mathrm{mol}$. The small differences can arise from the different choice of the basis sets used to obtained these MAEs. These results also indicate that variations in the scaling factor are not too crucial for the properties studied here. Full variational calculation will be implemented in future. Thus, the orbital SIC energies are scaled down as Eq. (4) or Eq. (7), and self-interaction correction to the Hamiltonian matrix elements of $i^{\text {th }}$ orbital are scaled down accordingly as Eq. (8) by ignoring the last two terms.

The orbital scaling calculations performed this way has comparable computational cost as PZSIC. The only additional cost is the calculation of the scaling factor which is not significant. For SCAN calculations, the FODs used in this study were optimized at the FLOSIC-SCAN level of theory where a convergence tolerance of at least $10^{-6}$ Ha was used. For all orbital scaling calculations, the FOD positions and electron densities optimized at the FLOSIC-SCAN level of theory were used as a starting point for a given system. For the AE6, BH6, SIE4 $\times 4$, and SIE11 calculations, we used the geometries from the test sets as provided. The SIE $4 \times 4$ calculations required us to use simple mixing with a larger mixing parameter $\alpha=0.15-0.35$ to achieve SCF convergence. OSIC-LSDA calculations were performed self-consistently in a similar fashion where we used FLOSIC-LSDA FODs and densities as a starting point.

Figure 1 shows the values of the scaling factors $X_{i \sigma}^{k}$ for $\mathrm{Kr}$ atom and benzene within 
OSIC-SCAN calculations. In both cases, core orbitals tend to have a larger value than the rest. For the case of benzene, the factors for C-C $\sigma$ bonds and $\pi$ bonds have the values less than 0.5. The actual values of the OSIC scaling factor depend on two elements: (i) compactness of local orbital and (ii) size of the single orbital regions identified from the iso-orbital indicator. For instance, the scaling factor of core orbitals in benzene is larger than that of 1s orbital in Kr atom as can be seen from the contour maps of the scaling factors in Fig. 2,

\section{RESULTS}

\section{A. Atoms: total energies, ionization potentials, and electron affinities}

We studied atoms $Z=1-18$ and compared the total energies using different scaling powers on PZSIC and OSIC with accurate non relativistic calculated reference values obtained by Chakravorty et al.[99]. The total energy differences with respect to reference values are shown in Fig. 3 for various values of $k$. The PZSIC corresponds to $k=0$, and all $k \geq 1$ results shown are with the OSIC method. We include the recently reported DFA-SCAN and PZSIC-SCAN results [79] here for comparison. DFA-SCAN shows small MAE of 0.019 Ha, and correcting for self-interaction results in significantly over-corrected total energies (MAE, $0.147 \mathrm{Ha}$ ) with systematic increase in error with increase in number of electrons. For Ar, the SI-correction is approximately $0.46 \mathrm{Ha}$. We note that application of OSIC reduces the MAE and as the $k$ values increases the MAE becomes smaller. The results for different scaling powers are summarized in Table II. OSIC-SCAN with $k=1$ reduces the MAE from 0.147 to $0.069 \mathrm{Ha}$, whereas $k=3$ shows comparable performance with DFA-SCAN. The best total energies are obtained with $k=4$ with MAE of only 0.012 Ha. These results are

consistent with earlier reports [30] that increasing value of $k$ results in better total energies. A comparison with previously reported results show that OSIC-SCAN with $k=4$ gives atomic energies that are better than the previously reported OSIC-LSDA, OSIC-PBE, or OSIC-TPSS. The MAE per electron of atoms Li to Ar for OSIC-SCAN $k=4$ is $0.002 \mathrm{Ha}$ whereas the reported best MAEs for OSIC-LSDA $k=1$, OSIC-PBE $k=3$, and OSIC-TPSS $k=3$ are $0.004,0.007$, and 0.003 Ha respectively. 

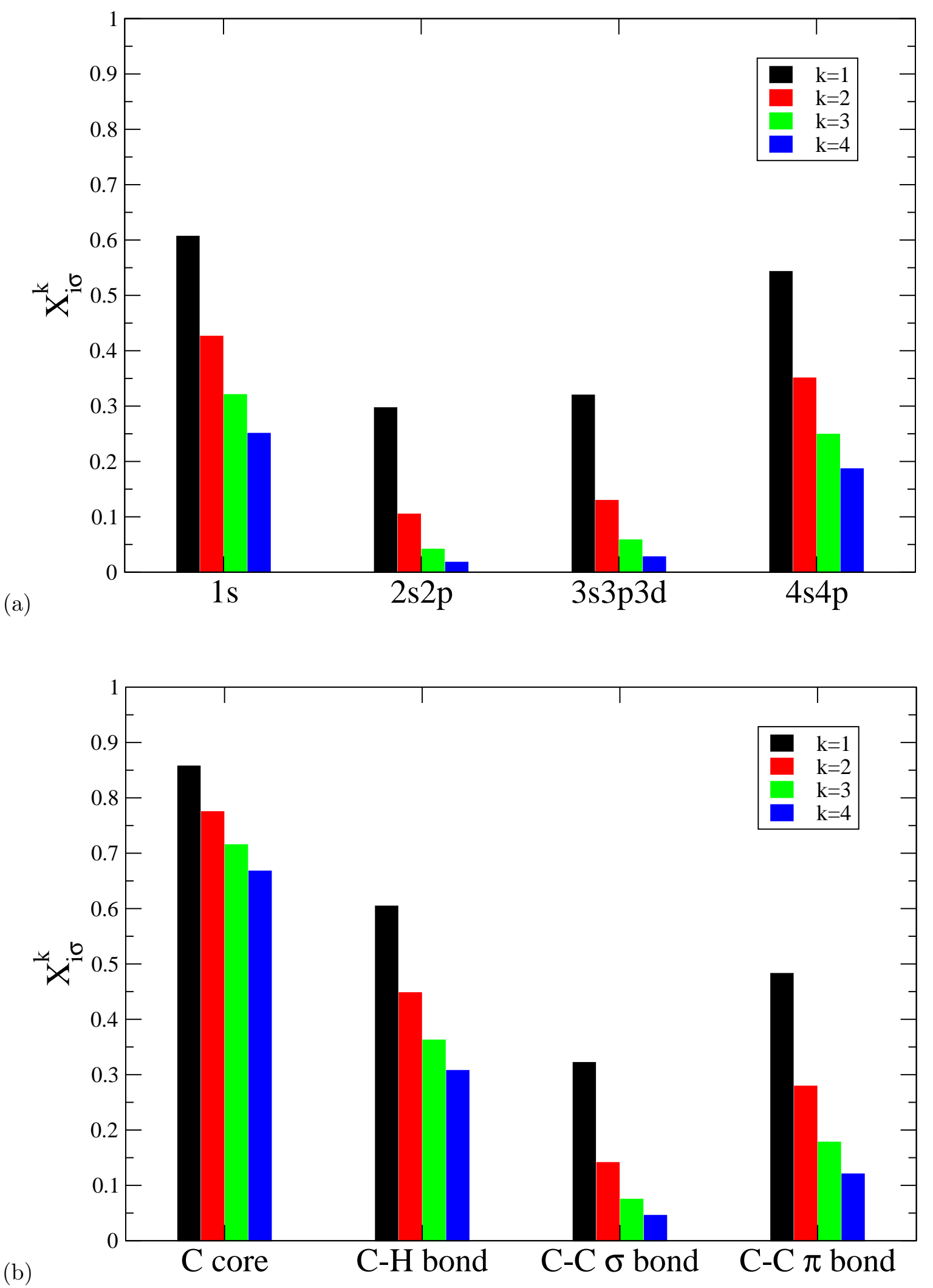

FIG. 1. Scaling factors $X_{i \sigma}^{k}$ with $z_{\sigma}(\vec{r})=\tau_{\sigma}^{W}(\vec{r}) / \tau_{\sigma}(\vec{r})$ and varying values of $k$ : (a) The averaged values for each electron shells of Kr atom and (b) the average values for each bond type of benzene. 


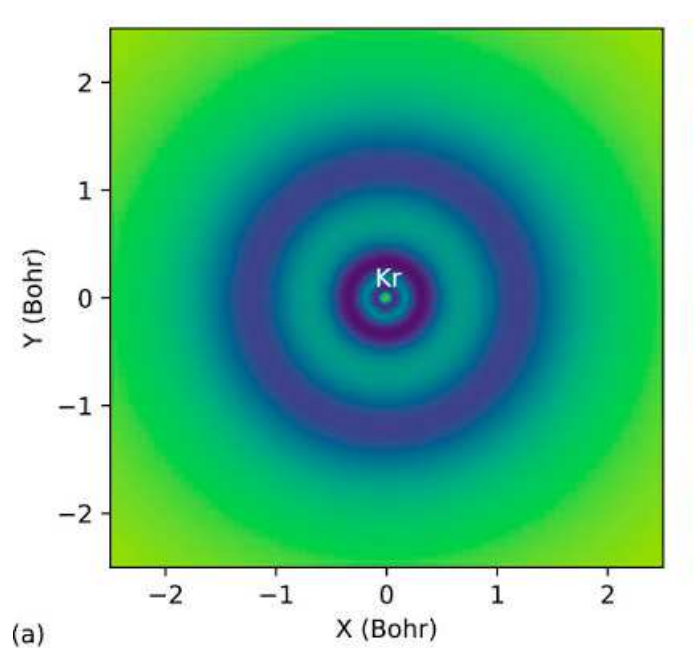

(a)

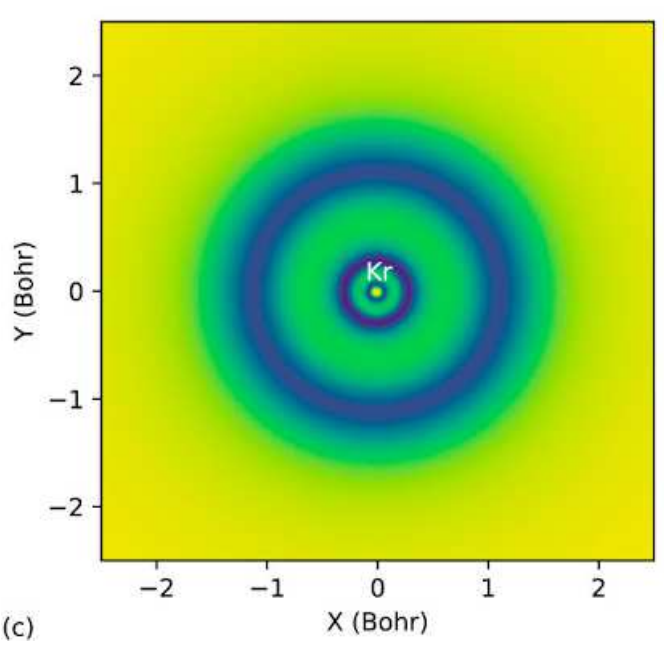

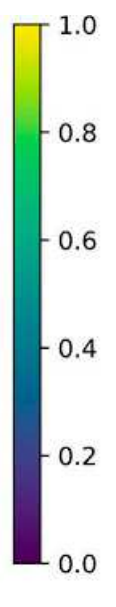
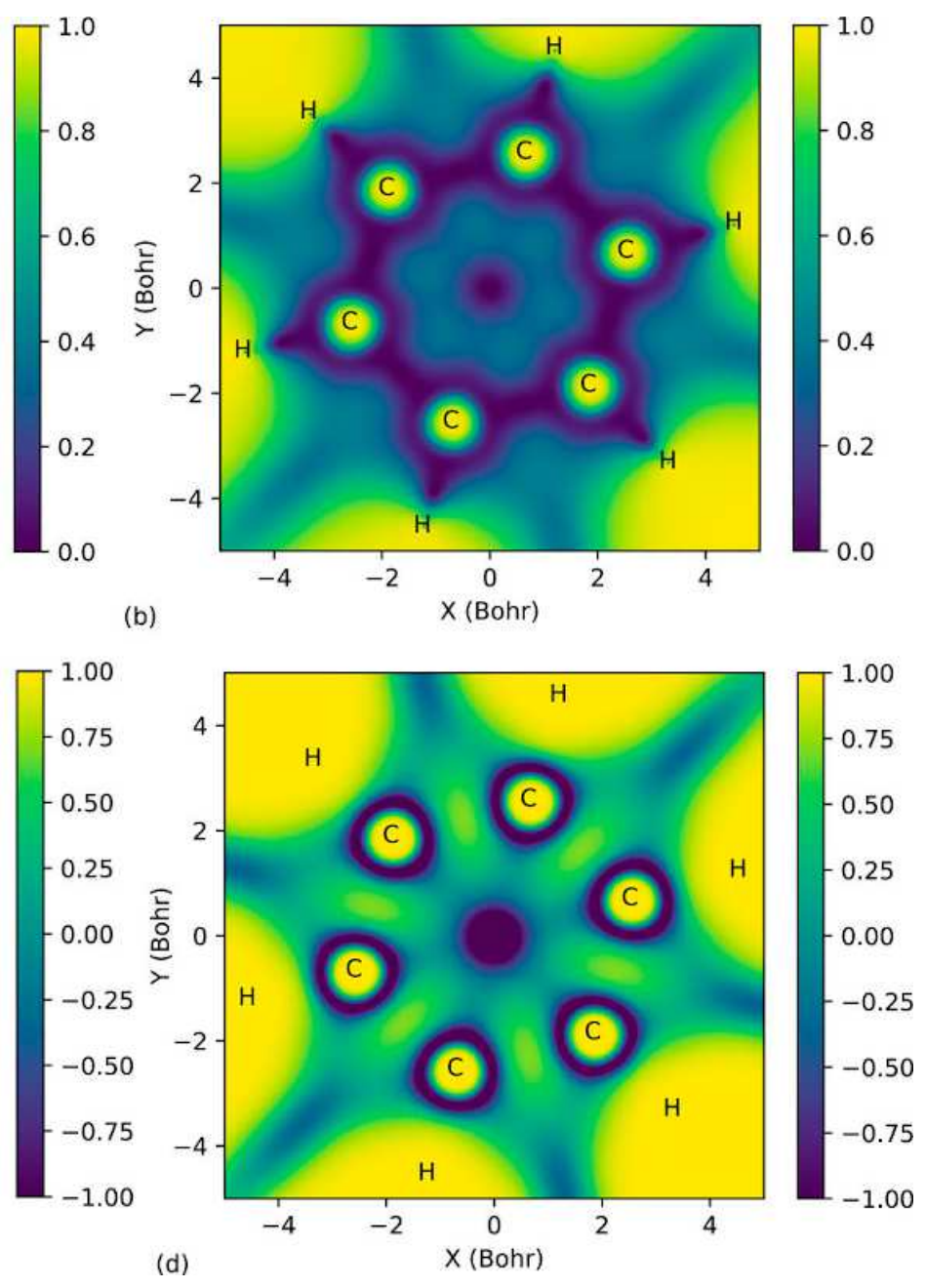

FIG. 2. The contour map of $z_{\sigma}(\vec{r})=\tau_{\sigma}^{W}(\vec{r}) / \tau_{\sigma}(\vec{r})$ for (a) Kr atom and (b) benzene in OSIC-SCAN $(k=1)$ calculations. Similarly, $1-(2 \beta)^{2}$ is shown for (c) Kr atom and (d) benzene. $z_{\sigma}(\vec{r})=1$ for the single orbital regions, and $z_{\sigma}(\vec{r})=0$ for the uniform density regions. For simplicity, only the spin-up kinetic energy density ratio is shown.

a. Ionization Potentials Ionization potentials (IP's) and electron affinities (EA's) are determined by processes that involve electron removal and electron addition, respectively. These processes are therefore sensitive to the asymptotic structure of the effective potential. One would therefore expect that removal of self-interaction in DFAs would result in significant improvement in the quality of these quantities. In general, however it has been found that these quantities calculated as the difference of total energies of neutral and charge system are fairly accurately predicted by many DFAs. The calculation of IPs (EAs) from the self-consistent total energy differences of atoms and their cation (anion) is called $\Delta \mathrm{SCF}$ 


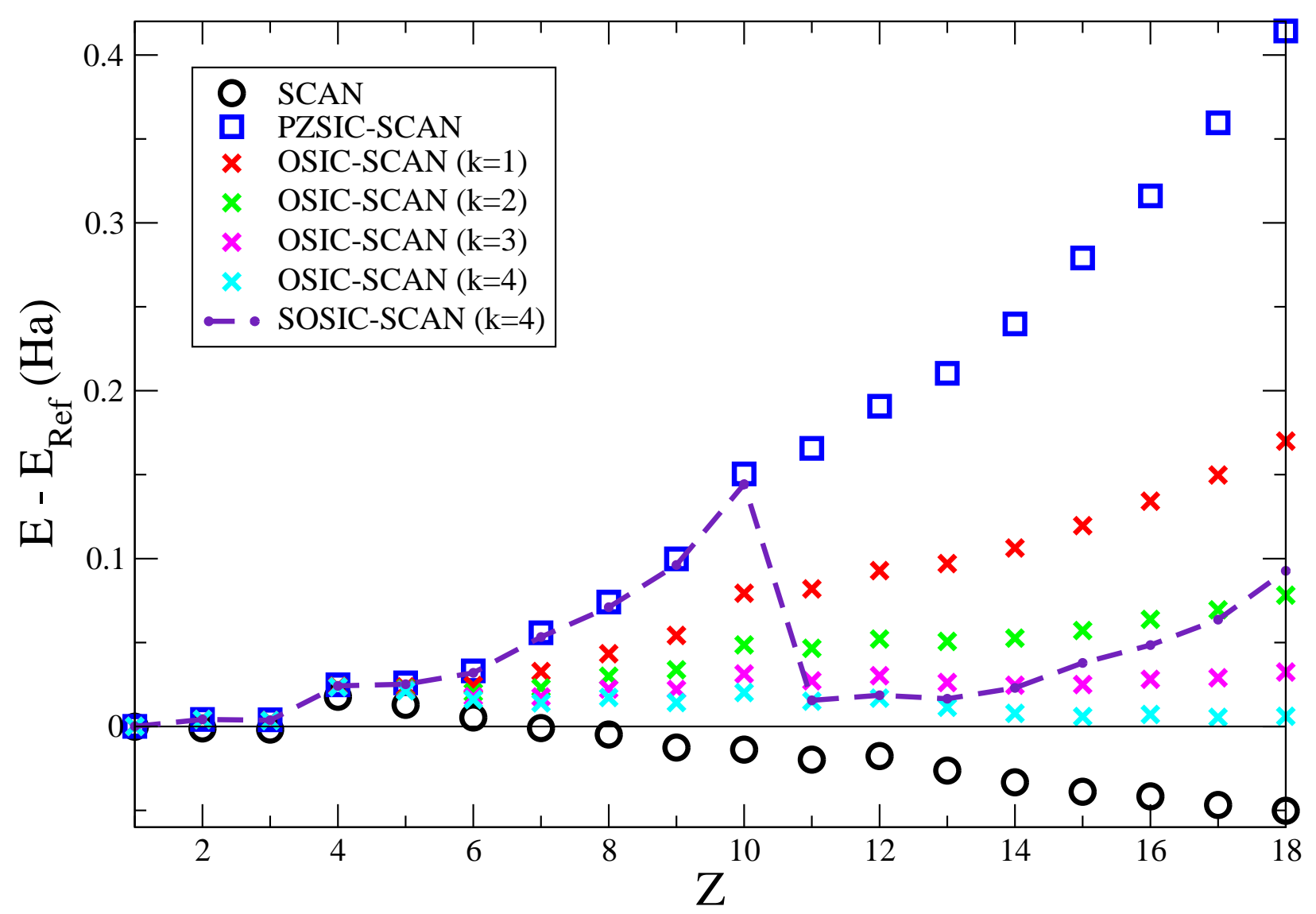

FIG. 3. Total energy difference (in Hartree) of atoms $\mathrm{Z}=1-18$ with respect to exact energies in various methods.

method[100]. We calculated the IPs for atoms from Hydrogen through Krypton using the $\triangle \mathrm{SCF}$ method and compared them against the experimentally reported values from Ref. [101] in Table II. To facilitate a direct comparison with the values reported by Vydrov et al., we also present results for a subset of atoms, from Hydrogen through Argon. For this smaller subset of atoms $Z=2-18$, the MAEs are 0.175 and $0.274 \mathrm{eV}$ for SCAN and PZSIC-SCAN respectively. The OSIC-SCAN results for various $k$ values have similar performance with MAEs within $0.178-0.181 \mathrm{eV}$. Vydrov et al. [30] reported that the OSIC with $k=2$ and 3 improves IPs of atoms $Z=1-18$ for LSDA, PBE, TPSS, and PBEh functionals. A similar trend was also observed in the present OSIC-SCAN ionization potentials for the subset of atoms. For this subset, the DFA already performs well and OSIC lowers the larger errors produced by PZSIC bringing the resultant errors close to those in DFA and in some cases improves them further. However, if one extends the data set to include a larger number of atoms $(Z=2-36)$, then a different trend is observed. In this case, PZSIC-SCAN (MAE 
$0.259 \mathrm{eV}$ ) shows better performance than DFA-SCAN (MAE $0.273 \mathrm{eV}$ ). The OSIC-SCAN results have MAEs ranging $0.304-0.349 \mathrm{eV}$, and these errors are larger than both DFA and PZSIC. This result suggests that full SIC treatment is needed to obtain accurate estimates of IPs of heavier atoms. All OSIC results for the complete set $(Z=2-36)$ studied here have similar errors as we have seen for the smaller subset $(Z=2-18)$, but there is a slight but noticeable decrease in errors as the value of $k$ increases.

b. Electron Affinity We studied EAs of 20 atoms, specifically atoms, H, Li, B, C, O, F, $\mathrm{Na}, \mathrm{Al}, \mathrm{Si}, \mathrm{P}, \mathrm{S}, \mathrm{Cl}, \mathrm{K}, \mathrm{Ti}, \mathrm{Cu}, \mathrm{Ga}, \mathrm{Ge}, \mathrm{As}, \mathrm{Se}$, and Br. These 20 atoms are experimentally shown to bind an extra electron, and their experimental EAs are found in the NIST database in Ref. [101]. Similar to the IP calculations, EAs were obtained using the $\triangle \mathrm{SCF}$ approach.

In Table III, we present results for a subset of 12 EAs for the first three rows of periodic table and the third column shows the results that include the fourth row in addition to the 12 EAs resulting in 20 EAs. For the 12 EAs, DFA-SCAN shows the smallest error but it has the problem of positive HOO eigenvalues. Correcting for SIE results in binding of the electron, and PZSIC-SCAN shows the MAE of $0.364 \mathrm{eV}$ for $\triangle \mathrm{SCF}$ EAs. The OSIC-SCAN with $k=4$ improves the EAs to MAE of $0.125 \mathrm{eV}$. For the larger set of 20 EAs, MAEs are 0.148 and $0.341 \mathrm{eV}$ for SCAN and PZSIC-SCAN in the respective order. The OSIC-SCAN gives performance improvement especially when $k=2$ is used. The error in this case is the smallest with MAE of $0.128 \mathrm{eV}$. Thus, OSIC-SCAN provides better performance for the EAs than the PZSIC-SCAN. We note that although the $\triangle \mathrm{SCF}$ approach yields positive EAs for the DFAs, the eigenvalue corresponding to the added electron becomes positive in all DFA anion calculations, indicating that the extra electron is not actually bound in the complete basis set limit. This problem is due to the incorrect asymptotic form of the potential in the DFA calculations. SIC fixes this [9], leading to bound states for the HOO in the anions. As mentioned in the introduction, the OSIC has undesirable effect on the asymptotic potential. In OSIC, the correct $-1 / r$ behavior of asymptotic potential in PZSIC is replaced by $-X_{H O} / r$ where $X_{H O}$ is the scaling factor for the electronic shell to which $\mathrm{HOO}$ belongs. In Fig. 4, we compared the HOO eigenvalues for PZSIC and OSIC calculations along with the experimental electron affinity. PZSIC gives negative HOO eigenvalues for all systems indicating the $\mathrm{HOO}$ electrons are bound to those atoms. It is evident from the figure that the absolute HOO eigenvalue in PZSIC overestimates the electron affinity. Applying OSIC shifts the eigenvalues upward. This upward shift for $k=1$ reduces the overestimation 
of absolute $\mathrm{HOO}$ as seen in PZSIC and bring it closer to the experimental electron affinities. But the shift systemically increases with the scaling factor as $k$ increases. As a consequence, the sign of the eigenvalue eventually changes for some systems and electron in HOO becomes unbound as the asymptotic potential becomes too shallow to provide sufficient attractive potential for the electron. This behavior was not noted earlier in the OSIC calculations of Vydrov and coworkers but it was expected as scaling down SIC by larger factors brings OSIC results closer to those of DFAs. The OSIC with $k=4$ has a drawback that several atomic anions are unbound in this model. Exceptions are alkali metals and halogens who maintained negative eigenvalues with OSIC unless very large scaling power $k$ is applied. These exceptions occur as halogens have larger electron affinities and because the scaled down factor for the HOO of alkali anions are large (e.g., 0.83 for $\mathrm{Li}^{-}$and 0.71 for $\mathrm{Na}^{-}$) even for $k=4$. For the rest of the atom families, anion HOO eigenvalues become positive with a scaling power of $2-3$. This is not too surprising considering OSIC recovers DFA performance in the $k \rightarrow \infty$ limit.

\section{B. Atomization energies}

The AE6 benchmark set [102] was used to study the performance of the OSIC approach in atomization energies. This set includes six molecules: $\mathrm{SiH}_{4}, \mathrm{SiO}, \mathrm{S}_{2}$, propyne $\left(\mathrm{C}_{3} \mathrm{H}_{4}\right)$, glyoxal $\left(\mathrm{C}_{2} \mathrm{H}_{2} \mathrm{O}_{2}\right)$, and cyclobutane $\left(\mathrm{C}_{4} \mathrm{H}_{8}\right)$, and it is a good representation of the larger main group atomization energy (MGAE109) set[103]. The geometries and reference values are obtained at the QCISD/MG3 level of theory. The atomization energy $(A E)$ of a molecule is obtained with

$$
A E=\sum_{i}^{N} E_{i}-E_{m o l}>0
$$

where $E_{i}$ is the energy of atom, $E_{m o l}$ is the energy of a given molecule, and $N$ is the number of atoms in the molecule. AEs were compared against non spin-orbit coupling reference values reported in Ref. [103]. The results are summarized in Table IV] The DFA-SCAN provides quite accurate estimates of $A E$ with MAE of only $2.85 \mathrm{kcal} / \mathrm{mol}$. However, correcting for SIE worsens AEs. PZSIC-LSDA FODs were used for the MAE reported in Ref. [69]. It is found that relaxation of FODs within PZSIC-SCAN increases the error (MAE 26.52 $\mathrm{kcal} / \mathrm{mol}$ ) using the data extracted from Ref. [79]. We observed that scaling down PZSIC improves the performance as the value of $k$ increases with $k=4$ yielding the best MAE 


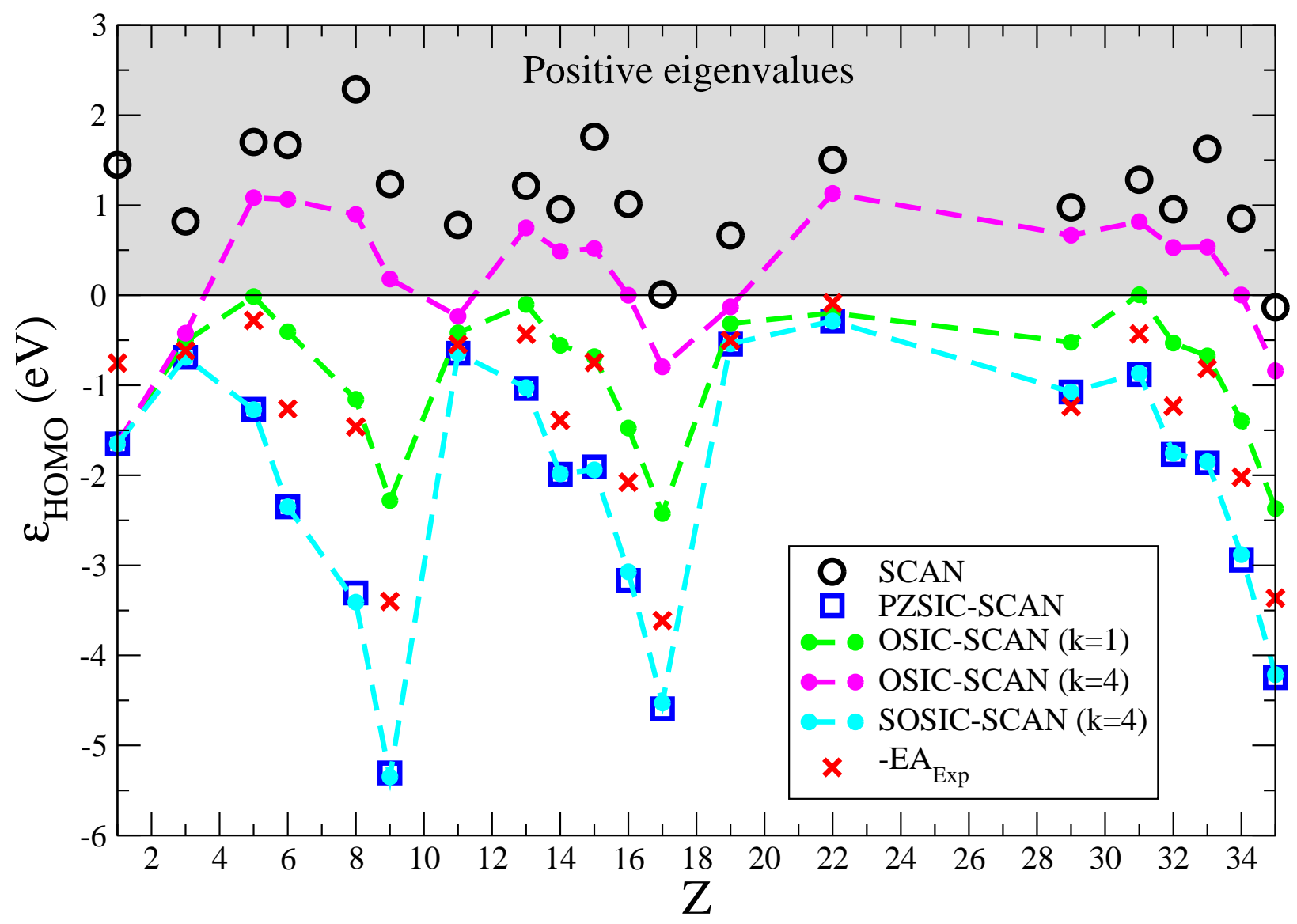

FIG. 4. The HOO eigenvalue of 20 atoms within various models along with negative of experimental EA values (in $\mathrm{eV})$.

of $4.10 \mathrm{kcal} / \mathrm{mol}$; although this error is larger in comparison than that of DFA-SCAN, the value is six times smaller compared to the PZSIC-SCAN result. PZSIC-SCAN tends to overestimate total energies especially for molecules, and this leads to a large discrepancy in atomization energies. Scaling down PZSIC helps reducing the overestimation and improves predicting atomization energies. This result shows that DFA-SCAN is better for predicting atomization energies without the self-interaction correction. Atomization energy calculations involve equilibrium molecular structure where the SCAN functional performs well.

\section{Reaction barrier heights}

BH6 benchmark set [102] was used to study the scaling down performance in reaction barrier. The BH6 set consists of three hydrogen transfer reactions $\left(\mathrm{OH}+\mathrm{CH}_{4} \rightarrow \mathrm{CH}_{3}+\right.$ 
$\mathrm{H}_{2} \mathrm{O}, \mathrm{H}+\mathrm{OH} \rightarrow \mathrm{O}+\mathrm{H}_{2}$, and $\left.\mathrm{H}+\mathrm{H}_{2} \mathrm{~S} \rightarrow \mathrm{H}_{2}+\mathrm{HS}\right)$. Total energies for the left- and right-hand side and saddle-point of a given reaction formula were calculated, and the barrier heights of forward (f) and reverse (r) reactions were obtained from the energy differences of these three points. Errors are summarized in Table V.

Many DFA functionals including SCAN do not give a correct picture of chemical reaction because in most cases the saddles points energies are underestimated. These are the cases where self-interaction correction becomes important. From the table it can be seen that PZSIC corrects the shortcoming of DFA in this situation. The full SIC treatment with $k=0$ reduces both ME and MAE. When orbital scaling is applied to PZSIC i.e., OSIC with $k=1$, the MAE increases to $3.96 \mathrm{kcal} / \mathrm{mol}$ from the PZSIC's $2.96 \mathrm{kcal} / \mathrm{mol}$. The MAE systematically increases with higher powers of the scaling factor. In all cases of OSIC, the reaction barriers are underestimated for all six reactions as can be seen from the MEs and MAEs in Table V]. For the saddle-point calculations with stretched bonds, one needs full $\mathrm{SIC}$ correction. The increase in value of $k$ results in larger percent of SIC correction being scaled down which leads to poor estimates of barrier heights. Note that for $k=4$, the error is comparable to the DFA error. A further discussion is presented in Sec. V]

\section{SIE benchmark sets}

The SIE11 sets consist of five cationic and six neutral chemical reactions that are very sensitive to self-interaction errors[104]. The SIE4 $\times 4$ sets consist of dissociation energy calculations of positively charged dimers at four different distances $R$ from their equilibrium distances $R_{e}: R / R_{e}=1.0,1.25,1.5$, and 1.75[105]. Reaction energies for SIE11 and dissociation energies for SIE4 $\times 4$ were computed and compared against the reference values. The dissociation energies and reaction energies are obtained from energy difference between left- and right-hand sides of a given chemical reaction formula. The reference values pro-

vided in Ref. [104] obtained at the coupled-cluster single double and perturbative triple $[\mathrm{CCSD}(\mathrm{T})] /$ CBS level of theory are used for comparison with our values. The results are presented in Table VI.

From SCAN to PZSIC-SCAN, there is substantial decrease in errors: for SIE $4 \times 4$, the MAE is decreased from 17.9 to $2.2 \mathrm{kcal} / \mathrm{mol}$. Similar performance improvements are also seen in the SIE11 test set where the MAEs decrease from 10.4 to $5.1 \mathrm{kcal} / \mathrm{mol}$ for the 
cationic reactions and from 9.9 to $6.2 \mathrm{kcal} / \mathrm{mol}$ for the neutral reactions. On the other hand, all of the OSIC results show larger errors compared to PZSIC-SCAN. Especially, for SIE4 $\times 4$ and SIE11 cationic reactions, larger MAEs are seen for higher $k$. For the SIE11 neutral systems, however, the error decreases for larger values of $k$ though it is still larger than the PZSIC-SCAN.

In our previous study [69], we used a pointwise local scaling approach on PZSIC-LSDA for the SIE sets. We found MAEs of 2.6, 2.31, and $6.31 \mathrm{kcal} / \mathrm{mol}$ for SIE4×4, SIE11 cationic, and SIE11 neutral reactions respectively. In all three cases, deviations were decreased from PZSIC-LSDA. In contrast, our OSIC results in Table VI show increase in errors going from PZSIC-SCAN to OSIC-SCAN. We find that orbital scaling does not perform well for the SIE4 $\times 4$ and SIE11 calculations, while LSIC [69], which is an interior scaling approach, does not experience the same performance degradation. The ideas to improve upon these shortcomings are discussed in Sec. V.

\section{PERFORMANCE OF DIFFERENT SCALING FACTORS}

In Table【, we compare the results of OSIC-LSDA with different iso-orbital indicators. For this comparison, we used $k=1$. We used ELF and $1-(2 \beta)^{2}$ as alternatives for the scaling factor in OSIC. We investigated the effect of these scaling factors in OSIC-LSDA and OSICSCAN on four different properties: total energies of atoms, atomization energies (AE6), barrier heights (BH6), and SIE sets of reactions. With OSIC-LSDA, $1-(2 \beta)^{2}$ produces larger MAE of $0.062 \mathrm{Ha}$ in the total energies of atoms compared to ELF (0.037 Ha) and $\tau^{W} / \tau(0.035 \mathrm{Ha})$. However, for the other properties, $1-(2 \beta)^{2}$ shows better performance than the others. For atomization energies, the factor $1-(2 \beta)^{2}$ yields an MAE of $11.7 \mathrm{kcal} / \mathrm{mol}$ compared to MAE of 23.2 and $18.9 \mathrm{kcal} / \mathrm{mol}$ for ELF and $\tau^{W} / \tau$, respectively. Similarly for barrier heights, $1-(2 \beta)^{2}$ (MAE, $2.3 \mathrm{kcal} / \mathrm{mol}$ ) shows better performance than ELF (MAE, $3.2 \mathrm{kcal} / \mathrm{mol}$ ) and $\tau^{W} / \tau$ (MAE, $\left.3.3 \mathrm{kcal} / \mathrm{mol}\right)$. A large difference can be seen for SIE11 where MAE is $5.9 \mathrm{kcal} / \mathrm{mol}$ for $1-(2 \beta)^{2}$. This is almost half of PZSIC-LSDA MAE of 11.7 $\mathrm{kcal} / \mathrm{mol}$ whereas the other two scaling factors show larger error than PZSIC-LSDA.

In addition to OSIC-LSDA, we also studied the effect of alternative scaling factors with OSIC-SCAN. For OSIC-SCAN, all three scaling factors have comparable performance in atomic total energies, AE6, and BH6. There are some differences for the SIE sets where 
ELF is similar to $\tau^{W} / \tau(k=3)$ and $1-(2 \beta)^{2}$ is similar to $\tau^{W} / \tau(k=2)$ in performance. Overall, the performance of PZSIC is best for the SIE sets of reactions.

\section{DISCUSSIONS AND IMPROVEMENTS TO THE OSIC}

Applications of OSIC in the present work to the SCAN functional show that the OSIC can overcome the worsening effects of the PZSIC results for equilibrium properties such as atomization energies or total energies if higher values of $k$ are used. For example, OSICSCAN with $k=4$ gives good total energies and atomization energies. On the other hand, The OSIC-SCAN with same $k=4$, results in deterioration of barrier heights or dissociation energies where the bonds are stretched. In this case, unscaled PZSIC (OSIC with $k=0$ ) works better than all scaled down PZSIC studied herein. Thus, no single value of $k$ is sufficient to obtain good results for all properties. These results are consistent with earlier scaled down PZSIC calculations of Vydrov and coworkers. The explanation as to why PZSIC does not perform well for semi-local functionals has been understood in terms of the orbital densities. It was shown that noded orbital densities produce large errors when used to estimate the self-interaction correction using Perdew-Zunger method[30, 106]. It was found that these errors can be reduced but not eliminated using nodeless densities of complex orbitals. Another source of error in PZSIC is that its application to a semilocal functional causes appropriate norms that are built in to the functional to be violated[107]. With OSIC, the loss of uniform electron gas limit depends on the form of the scaling factor used to identify many-electron region. As discussed in Sec. III, except for the ELF scaling factor used in this work, the OSIC has the correct uniform electron gas limit. The OSIC approach shows behavior that is opposite to the paradoxical behavior of original PZSIC. It improves some properties (equilibrium properties) at the cost of worsening the barrier heights where the bonds are stretched. Recent interior local scaling LSIC approach which corrects for the self-interaction in single-orbital region by scaling energy densities does not suffer from such conflicting behavior [69]. The OSIC thus has limited usefulness over PZSIC unless property dependent choice of $k$ (powers of scaling factor) is made. The external scaling form of OSIC (Eq. (4)) offers unique ways to apply the SIC (Eq. (7)). For example, the paradoxical behavior of OSIC can be mitigated by selectively applying the orbital scaling factor used in each local orbital. That is, one can apply the scaling with $k=4$ for most orbitals (core and 
part of valence states) and keep the full PZSIC correction for the orbitals that require full SIC treatment. We considered a few cases to demonstrate the potential of this approach which are discussed below.

\section{A. HOO eigenvalues}

One known shortcoming of orbital scaling is that the magnitude of the highest occupied eigenvalues $\left(\epsilon_{H O}\right)$ becomes underestimated. In exact DFT, the highest occupied eigenvalue equals the negative of the ionization potential[108-112]. This relationship does not strictly hold for approximate density functionals and in most DFAs, the absolute value of the HOO eigenvalue substantially underestimates the first ionization potential due to SIE. In Table VII, we compared MAEs of the HOO eigenvalues of atoms $Z=1-36$ against the experi-

mental IPs 101] using several different methods. PZSIC shows the smallest MAE of 0.606 $\mathrm{eV}$ as expected from the PZSIC's correct asymptotic potential shape, and the OSIC (scaled down PZSIC with $k>0$ ) generally shows larger deviations. This arises due to the scaling down the correction for the highest occupied orbital. The correct asymptotic behavior can be preserved if Eq. (7) is used instead of Eq. (4). To illustrate this, we applied the orbital scaling to PZSIC except for local orbitals on the electron shell that belong to the outermost electrons. The full PZSIC is used for these outermost orbitals. A comparison of the HOO eigenvalues of atoms so obtained are compared against experimental IPs[101] for a smaller subset of atoms with $Z=1-18$ is presented in Fig. 5. For this set, the OSIC with $k=4$ has an MAE of $2.414 \mathrm{eV}$ which is significantly larger compared to the PZSIC (MAE 0.763 $\mathrm{eV})$. On the other hand, SOSIC has MAE of only $0.754 \mathrm{eV}$ which shows that SOSIC can provide the $-\epsilon_{H O}$ of same quality as the PZSIC. It is interesting to see how the SOSIC affects total energies. We have shown this for atoms in Fig. 3 (SOSIC-SCAN $(k=4)$ ). Since orbitals other than those belong to HOO shell are scaled, the total energy in SOSIC would lie between OSIC $k=4$ and PZSIC total energies. Thus, lighter atoms for which most of the orbitals belong to the HOO shell have total energies closer to PZSIC. For benefiting both accuracy of PZSIC's $-\epsilon_{H O}$ and improved total energies from the orbital scaling, the best case is when a small fraction of local orbitals is mapped to HOO and is treated with full PZSIC. This is the case for the alkali metal atoms. In the worst case, SOSIC recovers the PZSIC energies. Halogens and noble gases atoms are the examples of such case (See 




FIG. 5. The difference in HOO eigenvalue of atoms $Z=1-18$ with respect to experimental IP (in eV). Note that, unlike OSIC eigenvalues, SOSIC eigenvalues closely mimic PZSIC eigenvalues.

Fig. 3).

\section{B. Barrier height}

Barrier height is another property that can benefit from selective scaling in OSIC. From Sec. III, we know that OSIC with $k=4$ give good atomization energies but poor barrier heights. The calculations of saddle points with stretched bonds are responsible for the increased discrepancies in the BH6 benchmark result. To see if barrier height estimates can be improved using selective scaling, we calculated the BH6 barrier heights using the following approach. Since we know that exterior scaling works well for the local orbitals in an equilibrium state, we applied the scaled down PZSIC $(k=4)$ for these orbitals while using full PZSIC for the orbital corresponding to the hydrogen transfer. With this selective scaling, we obtained MAE of $1.92 \mathrm{kcal} / \mathrm{mol}$ and $\mathrm{ME}$ of $-0.75 \mathrm{kcal} / \mathrm{mol}$. Curiously, this 
error is even smaller than MAE of $2.96 \mathrm{kcal} / \mathrm{mol}$ with PZSIC. This finding suggests that good results for barrier height calculation using the OSIC method can be achieved if scaling factors for certain orbitals are chosen according to the characteristics of orbitals as the spirit of SOSIC.

Finally, we comment on the possible effect of SOSIC on the dissociation energy curves. As mentioned in the introduction, Ruzsinszky and coworkers have studied the dissociation energy curves of $\mathrm{H}_{2}^{+}, \mathrm{He}_{2}^{+}, \mathrm{LiH}^{+}$, and $\mathrm{Ne}_{2}^{+}$using OSIC and have noted that unlike PZSIC, OSIC does not provide qualitatively correct curves for all four systems[74]. The SOSIC may correct this failure of OSIC as it provides correct asymptotic description of the potential. Our attempts to compute the dissociation curves for $\mathrm{LiH}^{+}$, and $\mathrm{Ne}_{2}^{+}$were not successful due to difficulties in obtaining convergence in far stretched regime using the Jacobi scheme of

Ref. [98]. A new method with a single SIC Hamiltonian is being developed[113] which shows promise in handling dissociating fractions with correct charge. We will study dissociation with SOSIC in future.

\section{CONCLUSIONS}

We have implemented the orbitalwise scaling down of PZSIC using the FLOSIC methodology. The OSIC method is used in combination with SCAN meta-GGA functional to assess its performance for a wide array of properties - for atoms: total energies, ionization potential, electron affinities, and for molecules: atomization energies, reaction barrier heights, and dissociation energies. We find that for equilibrium properties the OSIC with $k=4$ works well, and it recovers the performance of the uncorrected SCAN. For non-equilibrium properties, we observed that full PZSIC treatment is necessary in many situations. The comparison of present OSIC-SCAN results with earlier reported OSIC-PBE and OSICTPSS meta-GGA[30] indicate superior performance of OSIC-SCAN over the OSIC-PBE and OSIC-TPSS. We also show that by selectively scaling down and applying full PZSIC correction on active or outermost orbitals, the inconsistencies of OSIC can be mitigated or eliminated and its performance can be improved beyond equilibrium properties. Thus, selective scaling down approach presented here can provide good description of equilibrium properties, estimates of ionization energies from the HOO eigenvalues, stable atomic anions, and reaction barrier heights. The SOSIC thus provides major improvement over the OSIC 
formalism. It is interesting to compare the SOSIC approach with the LSIC method that we recently proposed[69]. The LSIC method removes the self-interaction selectively in spatial region where the correction is necessary and resolves the paradoxical behavior of PZSIC. It provides good results for both equilibrium properties as well as for properties where bonds are stretched. The SOSIC approach, though not as elegant as LSIC[69], accomplishes this goal by choosing the scaling factors according to the characteristic of orbitals. We hope

that the present results along with our recent results [69, 79] provide more sanguine future of SIC-DFA that has broader applicability than the standard DFAs.

\section{ACKNOWLEDGMENTS}

Authors acknowledge Profs. Koblar Jackson, Adrienn Ruzsinszky, Mark Pederson and John Perdew for comments on the manuscript. Discussions with Mr. Carlos Diaz and Dr. Luis Basurto are gratefully acknowledged. R. R. Z. is grateful to Prof. Rajeev K. Pathak for introducing him to self-interaction-corrected density functional theory. This work was supported by the US Department of Energy, Office of Science, Office of Basic Energy Sciences, as part of the Computational Chemical Sciences Program under Award No. de-sc0018331. Support for computational time at the Texas Advanced Computing Center through NSF Grant No. TG-DMR090071, and at NERSC is gratefully acknowledged.

\section{DATA AVAILABILITY STATEMENT}

The data that support the findings of this study are available from the corresponding author upon reasonable request. 


\section{TABLES}

TABLE I. The mean absolute error (MAE) of total atomic energies in various methods. These MAEs are in Hartree atomic unit.

\begin{tabular}{lcccc}
\hline \hline Method & LSDA & SCAN & PBE $^{\mathrm{a}, \mathrm{b}}$ & TPSS $^{\mathrm{a}, \mathrm{b}}$ \\
\hline DFA & $0.726(0.822)^{\mathrm{a}, \mathrm{b}}$ & 0.019 & 0.101 & 0.022 \\
PZSIC & $0.380(0.420)^{\mathrm{a}, \mathrm{b}}$ & 0.147 & 0.183 & 0.278 \\
OSIC $(k=1)$ & $0.035(0.037)^{\mathrm{a}, \mathrm{b}}$ & 0.069 & 0.118 & 0.131 \\
OSIC $(k=2)$ & $(0.205)^{\mathrm{a}, \mathrm{b}}$ & 0.038 & 0.095 & 0.073 \\
OSIC $(k=3)$ & $(0.316)^{\mathrm{a}, \mathrm{b}}$ & 0.021 & 0.085 & 0.042 \\
OSIC $(k=4)$ & & 0.012 & & \\
\hline SOSIC $(k=4)$ & & 0.043 & & \\
\hline OSIC $\left(z_{\sigma}=\mathrm{ELF}, k=1\right)$ & 0.037 & 0.069 & & \\
OSIC $\left[z_{\sigma}=1-(2 \beta)^{2}, k=1\right]$ & 0.062 & 0.063 & & \\
\hline LSIC & $0.041^{\mathrm{c}}$ & & \\
\hline \hline
\end{tabular}

a Reference [30].

b MAE reported in the reference is for atom $Z=3-18$.

c Reference [69]. 
TABLE II. The mean absolute error (in $\mathrm{eV}$ ) of $\triangle \mathrm{SCF}$ ionization potentials computed in various methods.

\begin{tabular}{|c|c|c|c|c|}
\hline \multirow[b]{2}{*}{ Method } & \multicolumn{2}{|c|}{ SCAN } & \multirow{2}{*}{$\frac{\mathrm{PBE}^{\mathrm{a}}}{\mathrm{Z}=1-18(18 \mathrm{IPs})}$} & \multirow{2}{*}{$\frac{\mathrm{TPSS}^{\mathrm{a}}}{\mathrm{Z}=1-18(18 \mathrm{IPs})}$} \\
\hline & $\mathrm{Z}=2-18(17 \mathrm{IPs})$ & $\mathrm{Z}=2-36(35 \mathrm{IPs})$ & & \\
\hline DFA & 0.175 & 0.273 & 0.15 & 0.12 \\
\hline PZSIC & 0.274 & 0.259 & 0.39 & 0.34 \\
\hline OSIC $(k=1)$ & 0.181 & 0.342 & 0.22 & 0.17 \\
\hline OSIC $(k=2)$ & 0.178 & 0.322 & 0.15 & 0.12 \\
\hline OSIC $(k=3)$ & 0.178 & 0.304 & 0.12 & 0.11 \\
\hline \multirow[t]{2}{*}{ OSIC $(k=4)$} & 0.183 & 0.294 & & \\
\hline & \multicolumn{2}{|c|}{ LSDA } & & \\
\hline Method & $\mathrm{Z}=2-18(17 \mathrm{IPs})$ & $\mathrm{Z}=2-36(35 \mathrm{IPs})$ & & \\
\hline LSIC $^{b}$ & 0.206 & 0.170 & & \\
\hline
\end{tabular}

a Reference 30 .

${ }^{\mathrm{b}}$ Reference [69]. 
TABLE III. The mean absolute error (in $\mathrm{eV}$ ) of $\triangle \mathrm{SCF}$ electron affinities computed in various methods.

\begin{tabular}{|c|c|c|c|c|}
\hline \multirow[b]{2}{*}{ Method } & \multicolumn{2}{|c|}{ SCAN } & \multirow{2}{*}{$\frac{\mathrm{PBE}^{\mathrm{a}}}{12 \mathrm{EAs}}$} & \multirow{2}{*}{$\frac{\mathrm{TPSS}^{\mathrm{a}}}{12 \mathrm{EAs}}$} \\
\hline & 12 EAs & $20 \mathrm{EAs}$ & & \\
\hline $\mathrm{DFA}^{\mathrm{b}}$ & 0.115 & 0.148 & 0.13 & 0.05 \\
\hline PZSIC & 0.364 & 0.341 & 0.57 & 0.47 \\
\hline OSIC $(k=1)$ & 0.198 & 0.151 & 0.29 & 0.24 \\
\hline OSIC $(k=2)$ & 0.143 & 0.128 & 0.15 & 0.12 \\
\hline OSIC $(k=3)$ & 0.126 & 0.134 & 0.10 & 0.08 \\
\hline \multirow[t]{2}{*}{ OSIC $(k=4)$} & 0.125 & 0.143 & & \\
\hline & \multicolumn{2}{|c|}{ LSDA } & & \\
\hline Method & $12 \mathrm{EAs}$ & 20 EAs & & \\
\hline $\mathrm{LSIC}_{-}$ & 0.097 & 0.102 & & \\
\hline
\end{tabular}

a Reference [30].

b DFA results are based on $\triangle \mathrm{SCF}$. The eigenvalue of an extra electron becomes positive (See text for details).

c Reference [69]. 
TABLE IV. The mean absolute and mean absolute percentage errors of AE6 set of molecules in various methods.

\begin{tabular}{|c|c|c|c|c|c|c|c|c|}
\hline \multirow[b]{2}{*}{ Method } & \multicolumn{2}{|c|}{ LSDA } & \multicolumn{2}{|c|}{ SCAN } & \multicolumn{2}{|c|}{$\mathrm{PBE}^{\mathrm{a}}$} & \multicolumn{2}{|c|}{ TPSS $^{\mathrm{a}}$} \\
\hline & $\begin{array}{c}\text { MAE } \\
(\mathrm{kcal} / \mathrm{mol})\end{array}$ & $\begin{array}{c}\text { MAPE } \\
(\%)\end{array}$ & $\begin{array}{c}\text { MAE } \\
(\mathrm{kcal} / \mathrm{mol})\end{array}$ & $\begin{array}{c}\text { MAPE } \\
(\%)\end{array}$ & $\begin{array}{c}\text { MAE } \\
(\mathrm{kcal} / \mathrm{mol})\end{array}$ & $\begin{array}{c}\text { MAPE } \\
(\%)\end{array}$ & $\begin{array}{c}\text { MAE } \\
(\mathrm{kcal} / \mathrm{mol})\end{array}$ & $\begin{array}{c}\text { MAPE } \\
(\%)\end{array}$ \\
\hline DFA & $74.26(77.3)^{\mathrm{a}}$ & $15.93(17.27)^{\mathrm{a}}$ & 2.85 & 1.15 & 15.5 & 4.43 & 5.9 & 2.43 \\
\hline PZSIC & $57.97(60.3)^{\mathrm{a}}$ & $9.37(10.61)^{\mathrm{a}}$ & 26.52 & 7.35 & 17.0 & 5.54 & 34.7 & 9.29 \\
\hline OSIC $(k=2)$ & $(8.6)^{\mathrm{a}}$ & $(3.56)^{\mathrm{a}}$ & 4.86 & 2.12 & 16.0 & 4.76 & 11.3 & 4.09 \\
\hline OSIC $(k=3)$ & $(7.2)^{\mathrm{a}}$ & $(3.41)^{\mathrm{a}}$ & 4.18 & 1.93 & 17.2 & 5.20 & 12.4 & 4.10 \\
\hline OSIC $(k=4)$ & & & 4.10 & 1.85 & & & & \\
\hline
\end{tabular}

\footnotetext{
${ }^{a}$ Reference 30.

b Reference [69].
} 
TABLE V. The mean and mean absolute errors (in kcal/mol) in barrier heights of BH6 set of molecules.

\begin{tabular}{|c|c|c|c|c|c|c|c|c|}
\hline \multirow[b]{2}{*}{ Method } & \multicolumn{2}{|c|}{ LSDA } & \multicolumn{2}{|c|}{ SCAN } & \multicolumn{2}{|c|}{$\mathrm{PBE}^{\mathrm{a}}$} & \multicolumn{2}{|c|}{ TPSS $^{\mathrm{a}}$} \\
\hline & $\mathrm{ME}$ & MAE & $\mathrm{ME}$ & MAE & $\mathrm{ME}$ & MAE & ME & MAE \\
\hline DFA & $-17.62(-17.9)^{\mathrm{a}}$ & $17.62(17.9)^{\mathrm{a}}$ & -7.86 & 7.86 & -9.5 & 9.5 & -8.5 & 8.5 \\
\hline PZSIC & $-4.88(-5.2)^{\mathrm{a}}$ & $4.88(5.2)^{\mathrm{a}}$ & -0.81 & 2.96 & -0.1 & 4.2 & -0.2 & 5.7 \\
\hline OSIC $(k=1)$ & $-2.95(-3.2)^{\mathrm{a}}$ & $3.31(3.5)^{\mathrm{a}}$ & -3.96 & 3.96 & -4.2 & 4.3 & -4.6 & 5.0 \\
\hline OSIC $(k=2)$ & $(-2.8)^{\mathrm{a}}$ & $(4.7)^{\mathrm{a}}$ & -5.45 & 5.45 & -6.5 & 6.5 & -6.8 & 6.8 \\
\hline OSIC $(k=3)$ & $(-2.9)^{\mathrm{a}}$ & $(5.7)^{\mathrm{a}}$ & -6.22 & 6.22 & -7.7 & 7.7 & -7.9 & 7.9 \\
\hline OSIC $(k=4)$ & & & -6.66 & 6.66 & & & & \\
\hline $\operatorname{SOSIC}(k=4) \stackrel{\mathrm{c}}{-}$ & & & -0.75 & 1.92 & & & & \\
\hline OSIC $\left(z_{\sigma}=\mathrm{ELF}, k=1\right)$ & -3.08 & 3.15 & -4.25 & 4.25 & & & & \\
\hline OSIC $\left[z_{\sigma}=1-(2 \beta)^{2}, k=1\right]$ & -1.88 & 2.28 & -4.87 & 4.87 & & & & \\
\hline $\mathrm{LSIC}^{\mathrm{b}}$ & 0.7 & 1.3 & & & & & & \\
\hline
\end{tabular}

${ }^{\text {a }}$ Reference 30 .

b Reference [69].

${ }^{c}$ Full PZSIC was applied on H-transfer FLOs (See text for details.) 
TABLE VI. The mean absolute error (in kcal/mol) of SIE $4 \times 4$ and SIE11 sets of molecules.

\begin{tabular}{lcccc}
\hline \hline Method & SIE4 $\times 4$ & SIE11 & SIE11, 5 cationic & SIE11, 6 neutral \\
\hline SCAN & 17.9 & 10.1 & 10.4 & 9.9 \\
PZSIC-SCAN & 2.2 & 5.7 & 5.1 & 6.2 \\
OSIC-SCAN $(k=1)$ & 2.9 & 14.7 & 6.5 & 21.5 \\
OSIC-SCAN $(k=2)$ & 5.2 & 13.4 & 7.6 & 18.2 \\
OSIC-SCAN $(k=3)$ & 6.5 & 7.4 & 7.7 & 7.0 \\
OSIC-SCAN $(k=4)$ & 7.4 & 8.5 & 7.7 & 9.2 \\
\hline OSIC-LSDA $\left(z_{\sigma}=\tau^{W} / \tau, k=1\right)$ & 4.7 & 15.4 & 9.4 & 20.4 \\
OSIC-LSDA $\left(z_{\sigma}=\mathrm{ELF}, k=1\right)$ & 7.3 & 18.0 & 11.8 & 23.1 \\
OSIC-LSDA $\left[z_{\sigma}=1-(2 \beta)^{2}, k=1\right]$ & 4.6 & 5.9 & 5.0 & 6.7 \\
\hline OSIC-SCAN $\left(z_{\sigma}=\mathrm{ELF}, k=1\right)$ & 5.0 & 7.3 & 6.4 & 7.9 \\
OSIC-SCAN $\left[z_{\sigma}=1-(2 \beta)^{2}, k=1\right]$ & 4.5 & 15.3 & 9.6 & 20.1 \\
\hline LSIC-LSDA & 2.6 & 4.5 & 2.3 & 6.3 \\
\hline \hline
\end{tabular}

a Reference [69].

TABLE VII. The mean absolute errors (in $\mathrm{eV})$ in the highest occupied eigenvalues $\left(-\epsilon_{H O}\right)$ for atoms hydrogen through argon and hydrogen through krypton.

\begin{tabular}{lcc}
\hline \hline Method & $Z=1-18 \mathrm{MAE}$ & $Z=1-36 \mathrm{MAE}$ \\
\hline SCAN & 4.549 & 3.880 \\
PZSIC-SCAN & 0.763 & 0.606 \\
OSIC-SCAN $(k=1)$ & 1.051 & 1.045 \\
OSIC-SCAN $(k=2)$ & 1.750 & 1.644 \\
OSIC-SCAN $(k=3)$ & 2.151 & 1.981 \\
OSIC-SCAN $(k=4)$ & 2.414 & 2.205 \\
\hline SOSIC-SCAN $(k=4)$ & 0.754 & \\
\hline \hline
\end{tabular}


[1] W. Kohn and L. Sham, Self-consistent equations including exchange and correlation effects, Phys. Rev. 140, A1133 (1965).

[2] P. Hohenberg and W. Kohn, Inhomogeneous electron gas, Phys. Rev. 136, B864 (1964).

[3] J. P. Perdew and K. Schmidt, Jacobs ladder of density functional approximations for the exchange-correlation energy, in AIP Conference Proceedings, Vol. 577 (AIP, 2001) pp. 1-20.

[4] J. P. Perdew and Y. Wang, Accurate and simple analytic representation of the electron-gas correlation energy, Phys. Rev. B 45, 13244 (1992).

[5] J. P. Perdew, K. Burke, and M. Ernzerhof, Generalized gradient approximation made simple, Phys. Rev. Lett. 77, 3865 (1996).

[6] J. P. Perdew, K. Burke, and M. Ernzerhof, Generalized gradient approximation made simple [phys. rev. lett. 77, 3865 (1996)], Phys. Rev. Lett. 78, 1396 (1997).

[7] A. D. Becke, A new mixing of Hartree-Fock and local density-functional theories, J. Chem. Phys. 98, 1372 (1993), https://doi.org/10.1063/1.464304.

[8] J. Gräfenstein, E. Kraka, and D. Cremer, The impact of the self-interaction error on the density functional theory description of dissociating radical cations: Ionic and covalent dissociation limits, J. Chem. Phys. 120, 524 (2004), https://doi.org/10.1063/1.1630017.

[9] J. P. Perdew and A. Zunger, Self-interaction correction to density-functional approximations for many-electron systems, Phys. Rev. B 23, 5048 (1981).

[10] Y. Zhang and W. Yang, A challenge for density functionals: Self-interaction error increases for systems with a noninteger number of electrons, The Journal of chemical physics 109, 2604 (1998).

[11] A. J. Cohen, P. Mori-Snchez, and W. Yang, Development of exchangecorrelation functionals with minimal many-electron self-interaction error, The Journal of Chemical Physics 126, 191109 (2007), https://doi.org/10.1063/1.2741248.

[12] D. S. Ranasinghe, J. T. Margraf, Y. Jin, and R. J. Bartlett, Does the ionization potential condition employed in QTP functionals mitigate the self-interaction error?, The Journal of Chemical Physics 146, 034102 (2017).

[13] P. Klüpfel, P. M. Dinh, P.-G. Reinhard, and E. Suraud, Koopmans condition in selfinteraction-corrected density-functional theory, Physical Review A 88, 052501 (2013). 
[14] N. I. Gidopoulos and N. N. Lathiotakis, Constraining density functional approximations to yield self-interaction free potentials, The Journal of Chemical Physics 136, 224109 (2012).

[15] R. A. Heaton, J. G. Harrison, and C. C. Lin, Self-interaction correction for density-functional theory of electronic energy bands of solids, Phys. Rev. B 28, 5992 (1983).

[16] M. R. Pederson, R. A. Heaton, and C. C. Lin, Local-density Hartree-Fock theory of electronic states of molecules with self-interaction correction, J. Chem. Phys. 80, 1972 (1984), https://doi.org/10.1063/1.446959.

[17] M. R. Pederson, R. A. Heaton, and C. C. Lin, Density-functional theory with self-interaction correction: Application to the lithium molecule), J. Chem. Phys. 82, 2688 (1985), https://doi.org/10.1063/1.448266.

[18] J. Garza, J. A. Nichols, and D. A. Dixon, The optimized effective potential and the self-interaction correction in density functional theory: Application to molecules, J. Chem. Phys. 112, 7880 (2000), https://doi.org/10.1063/1.481421.

[19] J. Garza, R. Vargas, J. A. Nichols, and D. A. Dixon, Orbital energy analysis with respect to LDA and self-interaction corrected exchange-only potentials, J. Chem. Phys. 114, 639 (2001), https://aip.scitation.org/doi/pdf/10.1063/1.1327269.

[20] S. Patchkovskii, J. Autschbach, and T. Ziegler, Curing difficult cases in magnetic properties prediction with self-interaction corrected density functional theory, J. Chem. Phys. 115, 26 (2001), https://doi.org/10.1063/1.1370527.

[21] M. K. Harbola, Theoretical investigation of the polarizability of small metal clusters, Solid State Commun. 98, 629 (1996).

[22] S. Patchkovskii and T. Ziegler, Improving "difficult" reaction barriers with selfinteraction corrected density functional theory, J. Chem. Phys. 116, 7806 (2002), https://doi.org/10.1063/1.1468640.

[23] S. Patchkovskii and T. Ziegler, Phosphorus NMR chemical shifts with selfinteraction free, gradient-corrected DFT, J. Phys. Chem. A 106, 1088 (2002), https://doi.org/10.1021/jp014184v

[24] S. Goedecker and C. J. Umrigar, Critical assessment of the self-interactioncorrected-local-density-functional method and its algorithmic implementation, Phys. Rev. A 55, 1765 (1997). 
[25] V. Polo, E. Kraka, and D. Cremer, Electron correlation and the selfinteraction error of density functional theory, Mol. Phys. 100,1771 (2002), https://doi.org/10.1080/00268970110111788.

[26] V. Polo, J. Gräfenstein, E. Kraka, and D. Cremer, Long-range and short-range coulomb correlation effects as simulated by Hartree-Fock, local density approximation, and generalized gradient approximation exchange functionals, Theor. Chim. Acta 109, 22 (2003).

[27] J. Gräfenstein, E. Kraka, and D. Cremer, Effect of the self-interaction error for three-electron bonds: On the development of new exchange-correlation functionals, Phys. Chem. Chem. Phys. 6, 1096 (2004).

[28] O. A. Vydrov and G. E. Scuseria, Effect of the Perdew-Zunger self-interaction correction on the thermochemical performance of approximate density functionals, J. Chem. Phys. 121, 8187 (2004), https://aip.scitation.org/doi/pdf/10.1063/1.1794633.

[29] O. A. Vydrov and G. E. Scuseria, Ionization potentials and electron affinities in the Perdew-Zunger self-interaction corrected density-functional theory, J. Chem. Phys. 122, 184107 (2005), https://doi.org/10.1063/1.1897378.

[30] O. A. Vydrov, G. E. Scuseria, J. P. Perdew, A. Ruzsinszky, and G. I. Csonka, Scaling down the Perdew-Zunger self-interaction correction in many-electron regions, J. Chem. Phys. 124, 094108 (2006), https://doi.org/10.1063/1.2176608.

[31] R. R. Zope, M. K. Harbola, and R. K. Pathak, Atomic Compton profiles within different exchange-only theories, Eur. Phys. J. D 7, 151 (1999).

[32] O. A. Vydrov and G. E. Scuseria, A simple method to selectively scale down the self-interaction correction, J. Chem. Phys. 124, 191101 (2006), https://doi.org/10.1063/1.2204599.

[33] T. Tsuneda, M. Kamiya, and K. Hirao, Regional self-interaction correction of density functional theory, J. Chem. Phys. 24, 1592 (2003), https://onlinelibrary.wiley.com/doi/pdf/10.1002/jcc.10279.

[34] J. B. Krieger, Y. Li, and G. J. Iafrate, Construction and application of an accurate local spin-polarized Kohn-Sham potential with integer discontinuity: Exchange-only theory, Phys. Rev. A 45, 101 (1992).

[35] J. B. Krieger, Y. Li, and G. J. Iafrate, Systematic approximations to the optimized effective potential: Application to orbital-density-functional theory, Phys. Rev. A 46, 5453 (1992). 
[36] U. Lundin and O. Eriksson, Novel method of self-interaction corrections in density functional calculations, Int. J. Quantum Chem. 81, 247 (2001).

[37] Y. Li, J. B. Krieger, and G. J. Iafrate, Self-consistent calculations of atomic properties using self-interaction-free exchange-only Kohn-Sham potentials, Phys. Rev. A 47, 165 (1993).

[38] S. Lehtola, M. Head-Gordon, and H. Jónsson, Complex orbitals, multiple local minima, and symmetry breaking in Perdew-Zunger self-interaction corrected density functional theory calculations, J. Chem. Theory Comput. 12, 3195 (2016).

[39] G. I. Csonka and B. G. Johnson, Inclusion of exact exchange for self-interaction corrected H3 density functional potential energy surface, Theor. Chim. Acta 99, 158 (1998).

[40] L. Petit, A. Svane, M. Lüders, Z. Szotek, G. Vaitheeswaran, V. Kanchana, and W. Temmerman, Phase transitions in rare earth tellurides under pressure, J. Phys. Cond. Matter 26, $274213(2014)$.

[41] S. Kümmel and L. Kronik, Orbital-dependent density functionals: Theory and applications, Rev. Mod. Phys. 80, 3 (2008).

[42] T. Schmidt, E. Kraisler, L. Kronik, and S. Kümmel, One-electron self-interaction and the asymptotics of the Kohn-Sham potential: an impaired relation, Phys. Chem. Chem. Phys. 16, 14357 (2014).

[43] D.-y. Kao, M. Pederson, T. Hahn, T. Baruah, S. Liebing, and J. Kortus, The role of selfinteraction corrections, vibrations, and spin-orbit in determining the ground spin state in a simple heme, Magnetochemistry 3, 31 (2017).

[44] S. Schwalbe, T. Hahn, S. Liebing, K. Trepte, and J. Kortus, Fermi-Löwdin orbital selfinteraction corrected density functional theory: Ionization potentials and enthalpies of formation, J. Comp. Chem. 39, 2463 (2018).

[45] H. Jónsson, K. Tsemekhman, and E. J. Bylaska, Accurate self-interaction correction to semilocal density functionals, in Abstracts of Papers of the American Chemical Society, Vol. 233 (American Chemical Society 1155 16TH ST, NW, Washington, DC 20036 USA, 2007) pp. $120-120$.

[46] M. M. Rieger and P. Vogl, Self-interaction corrections in semiconductors, Phys. Rev. B 52, 16567 (1995).

[47] W. Temmerman, A. Svane, Z. Szotek, H. Winter, and S. Beiden, On the implementation of the self-interaction corrected local spin density approximation for d-and f-electron systems, 
in Electronic Structure and Physical Properies of Solids (Springer, 1999) pp. 286-312.

[48] M. Daene, M. Lueders, A. Ernst, D. Ködderitzsch, W. M. Temmerman, Z. Szotek, and W. Hergert, Self-interaction correction in multiple scattering theory: application to transition metal oxides, J. Phys. Cond. Matter 21, 045604 (2009).

[49] Z. Szotek, W. Temmerman, and H. Winter, Self-interaction correction of localized bands within the LMTO-ASA band structure method, Physica B: Condensed Matter 172, 19 (1991).

[50] J. Messud, P. M. Dinh, P.-G. Reinhard, and E. Suraud, Time-dependent density-functional theory with a self-interaction correction, Phys. Rev. Lett. 101, 096404 (2008).

[51] J. Messud, P. M. Dinh, P.-G. Reinhard, and E. Suraud, Improved slater approximation to SIC-OEP, Chem. Phys. Lett. 461, 316 (2008).

[52] M. Lundberg and P. E. M. Siegbahn, Quantifying the effects of the self-interaction error in DFT: When do the delocalized states appear?, J. Chem. Phys. 122, 224103 (2005), https://doi.org/10.1063/1.1926277.

[53] T. Körzdörfer, M. Mundt, and S. Kümmel, Electrical response of molecular systems: the power of self-interaction corrected Kohn-Sham theory, Phys. Rev. Lett. 100, 133004 (2008).

[54] T. Körzdörfer, S. Kümmel, and M. Mundt, Self-interaction correction and the optimized effective potential, J. Chem. Phys. 129, 014110 (2008).

[55] I. Ciofini, C. Adamo, and H. Chermette, Self-interaction error in density functional theory: a mean-field correction for molecules and large systems, Chem. Phys. 309, 67 (2005).

[56] T. Baruah, R. R. Zope, A. Kshirsagar, and R. K. Pathak, Positron binding: A positrondensity viewpoint, Phys. Rev. A 50, 2191 (1994).

[57] A. I. Johnson, K. P. K. Withanage, K. Sharkas, Y. Yamamoto, T. Baruah, R. R. Zope, J. E. Peralta, and K. A. Jackson, The effect of self-interaction error on electrostatic dipoles calculated using density functional theory, J. Chem. Phys. 151, 174106 (2019), https://doi.org/10.1063/1.5125205.

[58] J. Vargas, P. Ufondu, T. Baruah, Y. Yamamoto, K. A. Jackson, and R. R. Zope, Importance of self-interaction-error removal in density functional calculations on water cluster anions, Phys. Chem. Chem. Phys., (2020).

[59] K. Trepte, S. Schwalbe, T. Hahn, J. Kortus, D.-Y. Kao, Y. Yamamoto, T. Baruah, R. R. Zope, K. P. K. Withanage, J. E. Peralta, and K. A. Jackson, Analytic atomic gradients in the Fermi-Löwdin orbital self-interaction correction, J. Comput. Chem. 40, 820 (2019), 
https://onlinelibrary.wiley.com/doi/pdf/10.1002/jcc.25767.

[60] K. P. K. Withanage, K. Trepte, J. E. Peralta, T. Baruah, R. Zope, and K. A. Jackson, On the question of the total energy in the Fermi-Löwdin orbital selfinteraction correction method, J. Chem. Theory Comput. 14, 4122 (2018), pMID: 29986131, https://doi.org/10.1021/acs.jctc.8b00344.

[61] M. R. Pederson, T. Baruah, D.-y. Kao, and L. Basurto, Self-interaction corrections applied to Mg-porphyrin, $\mathrm{C}_{60}$, and pentacene molecules, J. Chem. Phys. 144, 164117 (2016), https://doi.org/10.1063/1.4947042.

[62] S. Schwalbe, L. Fiedler, T. Hahn, K. Trepte, J. Kraus, and J. Kortus, PyFLOSIC - python based Fermi-Löwdin orbital self-interaction correction (2019), arXiv:1905.02631 [physics.comp-ph],

[63] D.-y. Kao, K. Withanage, T. Hahn, J. Batool, J. Kortus, and K. Jackson, Self-consistent self-interaction corrected density functional theory calculations for atoms using Fermi-Löwdin orbitals: Optimized Fermi-orbital descriptors for li-kr, J. Chem. Phys. 147, 164107 (2017), https://doi.org/10.1063/1.4996498.

[64] R. P. Joshi, K. Trepte, K. P. K. Withanage, K. Sharkas, Y. Yamamoto, L. Basurto, R. R. Zope, T. Baruah, K. A. Jackson, and J. E. Peralta, Fermi-Löwdin orbital self-interaction correction to magnetic exchange couplings, J. Chem. Phys. 149, 164101 (2018).

[65] K. Sharkas, L. Li, K. Trepte, K. P. K. Withanage, R. P. Joshi, R. R. Zope, T. Baruah, J. K. Johnson, K. A. Jackson, and J. E. Peralta, Shrinking self-interaction errors with the Fermi-Löwdin orbital self-interaction-corrected density functional approximation, J. Phys. Chem. A 122, 9307 (2018), pMID: 30412407.

[66] K. A. Jackson, J. E. Peralta, R. P. Joshi, K. P. Withanage, K. Trepte, K. Sharkas, and A. I. Johnson, Towards efficient density functional theory calculations without self-interaction: The Fermi-Löwdin orbital self-interaction correction, J. Phys. Conf. Ser. 1290, 012002 (2019),

[67] K. Sharkas, K. Wagle, B. Santra, S. Akter, R. R. Zope, T. Baruah, K. A. Jackson, J. P. Perdew, and J. E. Peralta, Self-interaction error overbinds water clusters but cancels in structural energy differences, Proc. Natl. Acad. Sci. To appear.

[68] J. P. Perdew, A. Ruzsinszky, J. Sun, and M. R. Pederson, Chapter one - paradox of self-interaction correction: How can anything so right be so wrong?, in 
Advances In Atomic, Molecular, and Optical Physics, Vol. 64 (Academic Press, 2015) pp. 1 -14 .

[69] R. R. Zope, Y. Yamamoto, C. M. Diaz, T. Baruah, J. E. Peralta, K. A. Jackson, B. Santra, and J. P. Perdew, A step in the direction of resolving the paradox of Perdew-Zunger self-interaction correction, J. Chem. Phys. 151, 214108 (2019), https://doi.org/10.1063/1.5129533.

[70] S. Klüpfel, P. Klüpfel, and H. Jónsson, The effect of the Perdew-Zunger selfinteraction correction to density functionals on the energetics of small molecules, J. Chem. Phys. 137, 124102 (2012).

[71] J. Tao, J. P. Perdew, V. N. Staroverov, and G. E. Scuseria, Climbing the density functional ladder: Nonempirical meta-generalized gradient approximation designed for molecules and solids, Phys. Rev. Lett. 91, 146401 (2003).

[72] C. Adamo and V. Barone, Toward reliable density functional methods without adjustable parameters: The PBE0 model, J. Chem. Phys. 110, 6158 (1999), https://doi.org/10.1063/1.478522.

[73] M. Ernzerhof and G. E. Scuseria, Assessment of the Perdew-Burke-Ernzerhof exchangecorrelation functional, J. Chem. Phys. 110, 5029 (1999), https://doi.org/10.1063/1.478401.

[74] A. Ruzsinszky, J. P. Perdew, G. I. Csonka, O. A. Vydrov, and G. E. Scuseria, Density functionals that are one- and two- are not always many-electron selfinteraction-free, as shown for $\mathrm{H}_{2}^{+}, \mathrm{He}_{2}^{+}, \mathrm{LiH}^{+}$, and $\mathrm{Ne}_{2}^{+}$, J. Chem. Phys. 126, 104102 (2007), https://doi.org/10.1063/1.2566637.

[75] J. Sun, A. Ruzsinszky, and J. P. Perdew, Strongly constrained and appropriately normed semilocal density functional, Phys. Rev. Lett. 115, 036402 (2015).

[76] Y. Fu and D. J. Singh, Applicability of the strongly constrained and appropriately normed density functional to transition-metal magnetism, Phys. Rev. Lett. 121, 207201 (2018).

[77] M. Chen, H.-Y. Ko, R. C. Remsing, M. F. C. Andrade, B. Santra, Z. Sun, A. Selloni, R. Car, M. L. Klein, J. P. Perdew, et al., Ab initio theory and modeling of water, Proceedings of the National Academy of Sciences 114, 10846 (2017).

[78] A. Paul, J. Sun, J. P. Perdew, and U. V. Waghmare, Accuracy of first-principles interatomic interactions and predictions of ferroelectric phase transitions in perovskite oxides: Energy functional and effective hamiltonian, Phys. Rev. B 95, 054111 (2017), 
[79] Y. Yamamoto, C. M. Diaz, L. Basurto, K. A. Jackson, T. Baruah, and R. R. Zope, Fermi-Löwdin orbital self-interaction correction using the strongly constrained and appropriately normed meta-GGA functional, J. Chem. Phys. 151, 154105 (2019), https://doi.org/10.1063/1.5120532.

[80] M. R. Pederson, A. Ruzsinszky, and J. P. Perdew, Communication: Self-interaction correction with unitary invariance in density functional theory, J. Chem. Phys. 140, 121103 (2014), https://doi.org/10.1063/1.4869581.

[81] W. L. Luken and D. N. Beratan, Localized orbitals and the Fermi hole, Theor. Chem. Acc. 61, 265 (1982).

[82] W. L. Luken and J. C. Culberson, Localized orbitals based on the Fermi hole, Theor. Chem. Acc. 66, 279 (1984).

[83] J. M. Leonard and W. L. Luken, Quadratically convergent calculation of localized molecular orbitals, Theor. Chem. Acc. 62, 107 (1982).

[84] D. C. Liu and J. Nocedal, On the limited memory BFGS method for large scale optimization, Math. Program. 45, 503 (1989).

[85] A. D. Becke and K. E. Edgecombe, A simple measure of electron localization in atomic and molecular systems, J. Chem. Phys. 92, 5397 (1990).

[86] J. W. Furness and J. Sun, Enhancing the efficiency of density functionals with an improved iso-orbital indicator, Phys. Rev. B 99, 041119 (2019).

[87] R. R. Zope and B. I. Dunlap, Slater's exchange parameters $\alpha$ for analytic and variational X $\alpha$ calculations, J. Chem. Theory Comput. 1, 1193 (2005).

[88] E. Livshits and R. Baer, A well-tempered density functional theory of electrons in molecules, Phys. Chem. Chem. Phys. 9, 2932 (2007).

[89] R. Baer, E. Livshits, and U. Salzner, Tuned range-separated hybrids in density functional theory, Annual review of physical chemistry 61, 85 (2010).

[90] L. Kronik, T. Stein, S. Refaely-Abramson, and R. Baer, Excitation gaps of finite-sized systems from optimally tuned range-separated hybrid functionals, J. Chem. Theory Comput. 8, 1515 (2012).

[91] M. E. Foster and B. M. Wong, Nonempirically tuned range-separated DFT accurately predicts both fundamental and excitation gaps in dna and rna nucleobases, J. Chem. Theory Comput. 8, 2682 (2012). 
[92] H. Sun and J. Autschbach, Influence of the delocalization error and applicability of optimal functional tuning in density functional calculations of nonlinear optical properties of organic donor-acceptor chromophores, ChemPhysChem 14, 2450 (2013).

[93] R. R. Zope, T. Baruah, Y. Yamamoto, L. Basurto, C. Diaz, J. Peralta, and K. A. Jackson, FLOSIC 0.1.2, based on the NRLMOL code of M. R. Pederson.

[94] Y. Yamamoto, L. Basurto, C. M. Diaz, R. R. Zope, and T. Baruah, Self-interaction correction to density functional approximations using Fermi-Löwdin orbitals: methodology and parallelization, unpublished.

[95] D. Porezag and M. R. Pederson, Optimization of gaussian basis sets for density-functional calculations, Phys. Rev. A 60, 2840 (1999).

[96] M. R. Pederson and K. A. Jackson, Variational mesh for quantum-mechanical simulations, Phys. Rev. B 41, 7453 (1990).

[97] K. P. K. Withanage, S. Akter, C. Shahi, R. P. Joshi, C. Diaz, Y. Yamamoto, R. Zope, T. Baruah, J. P. Perdew, J. E. Peralta, and K. A. Jackson, Self-interaction-free electric dipole polarizabilities for atoms and their ions using the Fermi-Löwdin self-interaction correction, Phys. Rev. A 100, 012505 (2019).

[98] Z.-h. Yang, M. R. Pederson, and J. P. Perdew, Full self-consistency in the Fermi-orbital self-interaction correction, Phys. Rev. A 95, 052505 (2017).

[99] S. J. Chakravorty, S. R. Gwaltney, E. R. Davidson, F. A. Parpia, and C. F. Fischer, Ground-state correlation energies for atomic ions with 3 to 18 electrons, Phys. Rev. A 47, 3649 (1993).

[100] O. Gunnarsson and B. I. Lundqvist, Exchange and correlation in atoms, molecules, and solids by the spin-density-functional formalism, Phys. Rev. B 13, 4274 (1976).

[101] National Institute of Standards and Technology, NIST Computational Chemistry Comparison and Benchmark Database NIST Standard Reference Database Number 101 Release 19, April 2018, Editor: Russell D. Johnson III http://cccbdb.nist.gov/ DOI:10.18434/T47C7Z.

[102] B. J. Lynch and D. G. Truhlar, Small representative benchmarks for thermochemical calculations, J. Phys. Chem. A 107, 8996 (2003), https://doi.org/10.1021/jp035287b.

[103] R. Peverati and D. G. Truhlar, Communication: A global hybrid generalized gradient approximation to the exchange-correlation functional that satisfies the second-order density-gradient 
constraint and has broad applicability in chemistry, J. Chem. Phys. 135, 191102 (2011), https://doi.org/10.1063/1.3663871.

[104] L. Goerigk and S. Grimme, A general database for main group thermochemistry, kinetics, and noncovalent interactions - assessment of common and reparameterized (meta-)GGA density functionals, J. Chem. Theory Comput. 6, 107 (2010), pMID: 26614324, https://doi.org/10.1021/ct900489g

[105] L. Goerigk, A. Hansen, C. Bauer, S. Ehrlich, A. Najibi, and S. Grimme, A look at the density functional theory zoo with the advanced GMTKN55 database for general main group thermochemistry, kinetics and noncovalent interactions, Phys. Chem. Chem. Phys. 19, 32184 (2017)

[106] C. Shahi, P. Bhattarai, K. Wagle, B. Santra, S. Schwalbe, T. Hahn, J. Kortus, K. A. Jackson, J. E. Peralta, K. Trepte, S. Lehtola, N. K. Nepal, H. Myneni, B. Neupane, S. Adhikari, A. Ruzsinszky, Y. Yamamoto, T. Baruah, R. R. Zope, and J. P. Perdew, Stretched or noded orbital densities and self-interaction correction in density functional theory, J. Chem. Phys. 150, 174102 (2019).

[107] B. Santra and J. P. Perdew, Perdew-Zunger self-interaction correction: How wrong for uniform densities and large-Z atoms?, J. Chem. Phys. 150, 174106 (2019), https://doi.org/10.1063/1.5090534.

[108] J. P. Perdew, R. G. Parr, M. Levy, and J. L. Balduz Jr, Density-functional theory for fractional particle number: derivative discontinuities of the energy, Phys. Rev. Lett. 49, 1691 (1982).

[109] M. Levy, J. P. Perdew, and V. Sahni, Exact differential equation for the density and ionization energy of a many-particle system, Phys. Rev. A 30, 2745 (1984).

[110] C.-O. Almbladh and U. von Barth, Exact results for the charge and spin densities, exchangecorrelation potentials, and density-functional eigenvalues, Phys. Rev. B 31, 3231 (1985).

[111] J. P. Perdew and M. Levy, Comment on "Significance of the highest occupied Kohn-Sham eigenvalue", Phys. Rev. B 56, 16021 (1997).

[112] M. K. Harbola, Relationship between the highest occupied Kohn-Sham orbital eigenvalue and ionization energy, Phys. Rev. B 60, 4545 (1999).

[113] M. R. Pederson and T. Baruah, Single Hamiltonian for self-interaction corrected DFT with Fermi-Löwdin orbitals, unpublished. 